\title{
FINITE ELEMENT APPROXIMATION OF THE CAHN-HILLIARD-COOK EQUATION
}

\author{
MIHÁLY KOVÁCS*, STIG LARSSON ${ }^{\dagger}$, AND ALI MESFORUSH
}

\begin{abstract}
We study the nonlinear stochastic Cahn-Hilliard equation perturbed by additive colored noise. We show almost sure existence and regularity of solutions. We introduce spatial approximation by a standard finite element method and prove error estimates of optimal order on sets of probability arbitrarily close to 1 . We also prove strong convergence without known rate.
\end{abstract}

Key words. Cahn-Hilliard-Cook equation, additive noise, Wiener process, existence, regularity, finite element, error estimate, strong convergence

AMS subject classifications. 65M60, 60H15, 60H35, 65C30

1. Introduction. We study the Cahn-Hilliard equation perturbed by noise, also known as the Cahn-Hilliard-Cook equation (cf. [2, 4]),

$$
\begin{array}{ll}
\mathrm{d} u-\Delta w \mathrm{~d} t=\mathrm{d} W & \text { in } \mathcal{D} \times(0, T], \\
w=-\Delta u+f(u) & \text { in } \mathcal{D} \times(0, T], \\
\frac{\partial u}{\partial n}=\frac{\partial w}{\partial n}=0 & \text { on } \partial \mathcal{D} \times(0, T], \\
u(0)=u_{0} & \text { in } \mathcal{D} .
\end{array}
$$

Here $\mathcal{D}$ is a bounded domain in $\mathbf{R}^{d}, d=1,2,3$, and $f(s)=s^{3}-s$. Using the framework of [6] we write this as an abstract evolution equation of the form

$$
\mathrm{d} X+\left(A^{2} X+A f(X)\right) \mathrm{d} t=\mathrm{d} W, \quad t \in(0, T] ; \quad X(0)=X_{0},
$$

where $A$ denotes the negative Neumann Laplacian considered as an unbounded operator in the Hilbert space $H=L_{2}(\mathcal{D})$ and $W$ is a $Q$-Wiener process in $H$ with respect to a filtered probability space $\left(\Omega, \mathcal{F}, \mathbf{P},\left\{\mathcal{F}_{t}\right\}_{t \geq 0}\right)$. We also write $H^{s}=H^{s}(\mathcal{D})$ for the standard Sobolev spaces. See Section 2 for details.

Our goal is to study the convergence properties of the spatially semidiscrete finite element approximation $X_{h}$ of $X$, which is defined by an equation of the form

$$
\mathrm{d} X_{h}+\left(A_{h}^{2} X_{h}+A_{h} P_{h} f\left(X_{h}\right)\right) \mathrm{d} t=P_{h} \mathrm{~d} W, \quad t \in(0, T] ; \quad X_{h}(0)=P_{h} X_{0} .
$$

In order to do so, we need to prove existence and regularity for solutions of (1.1). Such results were first proved in [5]. Under the assumption that the covariance operator $Q=I$ (space-time white noise, cylindrical noise) it was shown that there is a process which belongs to $C\left([0, T], H^{-1}\right)$ almost surely and which is the unique solution of 1.1). Under the stronger assumption that $A$ and $Q$ commute and that $\operatorname{Tr}\left(A^{\delta-1} Q\right)<$ $\infty$ for some $\delta>0$ (colored noise) it was shown that the solution belongs to $C([0, T], H)$ almost surely. Such regularity is insufficient for proving convergence of a numerical solution. Our first aim is therefore to prove existence of a solution in $C\left([0, T], H^{\beta}\right)$ almost surely for some $\beta>0$.

\footnotetext{
* Supported by a University of Otago Research Grant (UORG).

†Supported by the Swedish Research Council (VR) and by the Swedish Foundation for Strategic Research (SSF) through GMMC, the Gothenburg Mathematical Modelling Centre.
} 
Following the semigroup approach of [6] we write the equation 1.1 as the integral equation (mild solution)

$$
\begin{aligned}
X(t) & =\mathrm{e}^{-t A^{2}} X_{0}-\int_{0}^{t} A \mathrm{e}^{-(t-s) A^{2}} f(X(s)) \mathrm{d} s+\int_{0}^{t} \mathrm{e}^{-(t-s) A^{2}} \mathrm{~d} W(s) \\
& =Y(t)+W_{A}(t)
\end{aligned}
$$

where $\mathrm{e}^{-t A^{2}}$ is the analytic semigroup generated by $-A^{2}$ (see Corollary 4.2). This naturally splits the solution as $X=Y+W_{A}$, where $W_{A}(t)=\int_{0}^{t} \mathrm{e}^{-(t-s) A^{2}} \mathrm{~d} W(s)$ is a stochastic convolution. This convolution, and its finite element approximation, was studied in [12]. In particular, it was shown there that if $\left\|A^{\frac{\beta-2}{2}} Q^{\frac{1}{2}}\right\|_{\mathrm{HS}}^{2}<\infty$ for some $\beta \geq 0$, then we have regularity of order $\beta$ in a mean square sense; that is,

$$
\mathbf{E}\left[\left\|W_{A}(t)\right\|_{H^{\beta}}^{2}\right] \leq\left\|A^{\frac{\beta-2}{2}} Q^{\frac{1}{2}}\right\|_{\mathrm{HS}}^{2}, \quad t \geq 0 .
$$

The other part, $Y$, solves a differential equation with random coefficient,

$$
\dot{Y}+A^{2} Y+A f\left(Y+W_{A}\right)=0, \quad t>0 ; \quad Y(0)=X_{0} .
$$

This can be solved once $W_{A}$ is known. This approach was also used in [5], but while they used Galerkin's method and energy estimates to solve (1.3), we use a semigroup approach similar to that of [7. However, published results for the deterministic CahnHilliard equation do not apply directly due to the limited regularity in 1.3 .

The nonlinear term is only locally Lipschitz and we need to control the Lipschitz constant. In the deterministic case studied in [7] this is achieved by the Lyapunov functional

$$
J(u)=\frac{1}{2}\|\nabla u\|^{2}+\int_{\mathcal{D}} F(u) \mathrm{d} x, \quad u \in H^{1} ; \quad F(s)=\frac{1}{4} s^{4}-\frac{1}{2} s^{2},
$$

which is nonincreasing along paths, so that $\|X(t)\|_{H^{1}} \leq C$ for $t \geq 0$. Due to the stochastic perturbation, this is not true for the stochastic equation (1.1). However, it is possible find a bound for the growth of the expected value of $J(X(t))$,

$$
\mathbf{E}[J(X(t))] \leq C(t), \quad t \geq 0 .
$$

This was shown in [5] under the assumption that $A$ and $Q$ commute and

$$
\operatorname{Tr}(A Q)<\infty,
$$

which is consistent with $\beta=3$ in 1.2 , since $\left\|A^{\frac{1}{2}} Q^{\frac{1}{2}}\right\|_{\mathrm{HS}}^{2}=\operatorname{Tr}(A Q)$ in this case. (More generally: if $A Q$ is nuclear, then $\left\|A^{\frac{1}{2}} Q^{\frac{1}{2}}\right\|_{\mathrm{HS}}^{2}=\operatorname{Tr}(A Q)$, see 11, Theorem 2.1].) We repeat this in Theorem 3.1 with several improvements. First of all we reduce the growth of the bound from exponential to quadratic with respect to $t$. We also relax the assumptions: we do not assume that $A$ and $Q$ commute; that is, have a common eigenbasis, and we do not assume that the eigenbasis of $Q$ consists of bounded functions. Moreover, we prove the same bound for the finite element solution $X_{h}$. Even if $A$ and $Q$ commute, this will not be true for the corresponding finite element approximations $A_{h}$ and $Q_{h}$, so the relaxation of this assumption is necessary for the proof of the bound for $X_{h}$. 
In Corollary 3.2 we improve 1.4 to a uniform norm bound

$$
\mathbf{E}\left[\sup _{s \in[0, T]}\left(\|X(s)\|_{H^{1}}^{2}+\left\|X_{h}(s)\right\|_{H^{1}}^{2}\right)\right] \leq K_{T} .
$$

By means of Chebyshev's inequality we may then show that, for each $T>0$ and $\epsilon \in(0,1)$, there are $K_{T}$ and $\Omega_{\epsilon} \subset \Omega$ with $\mathbf{P}\left(\Omega_{\epsilon}\right) \geq 1-\epsilon$ and such that

$$
\|X(t)\|_{H^{1}}^{2}+\left\|X_{h}(t)\right\|_{H^{1}}^{2} \leq \epsilon^{-1} K_{T} \quad \text { on } \Omega_{\epsilon}, t \in[0, T] .
$$

This bound controls the Lipschitz constant of the nonlinear term and we show in Theorem 4.3 that $X \in C\left([0, T], H^{3}\right)$ for $\omega \in \Omega_{\epsilon}$ under the slightly stronger assumption that $\left\|A^{\frac{\gamma}{2}} Q^{\frac{1}{2}}\right\|_{\mathrm{HS}}<\infty$ for some $\gamma>1$, which is basically consistent with 1.5 and also with $[1.2$ in case $\beta=3$. We also obtain an error estimate (see Theorem 5.3)

$$
\left\|X_{h}(t)-X(t)\right\| \leq C\left(\epsilon^{-1} K_{T}, T\right) h^{2}|\log (h)| \quad \text { on } \Omega_{\epsilon}, t \in[0, T] .
$$

The constant grows rapidly with $\epsilon^{-1} K_{T}$, but nevertheless we may use this to show strong convergence (see Theorem 5.4,

$$
\mathbf{E}\left[\sup _{t \in[0, T]}\left\|X_{h}(t)-X(t)\right\|^{2}\right] \rightarrow 0 \quad \text { as } h \rightarrow 0 .
$$

To prove strong convergence with an estimate of the rate remains a challenge for future work. In this connection we note that even for numerical methods for stochastic ordinary differential equations with local Lipschitz nonlinearity there are few results on convergence rates (cf. [9]).

Numerical methods for the deterministic Cahn-Hilliard equation are well covered in the literature. There are few studies of numerical methods for the Cahn-HilliardCook equation. We are only aware of $[3$ in which convergence in probability was proved for a difference scheme for the nonlinear equation in multiple dimensions. For the linear equation there is [10], where strong convergence estimates were proved for the finite element method for the linear equation in 1-D, and the already mentioned work [12] on the finite element method for the stochastic convolution in multiple dimensions.

\section{Preliminaries.}

2.1. Norms. Let $\mathcal{D} \subset \mathbf{R}^{d}, d=1,2,3$, be a bounded convex domain with polygonal boundary $\partial \mathcal{D}$. Let $H=L_{2}(\mathcal{D})$ with standard inner product $\langle\cdot, \cdot\rangle$ and norm $\|\cdot\|$, and

$$
\dot{H}=\left\{v \in H: \int_{\mathcal{D}} v \mathrm{~d} x=0\right\} .
$$

Let $P: H \rightarrow \dot{H}$ define the orthogonal projector. Then

$$
(I-P) v=|\mathcal{D}|^{-1} \int_{\mathcal{D}} v \mathrm{~d} x,
$$

is the average of $v$. We also denote by $H^{k}=H^{k}(\mathcal{D})$ the standard Sobolev space. We define $A=-\Delta$ with domain of definition

$$
D(A)=\left\{v \in H^{2}: \frac{\partial v}{\partial n}=0 \text { on } \partial \mathcal{D}\right\} .
$$


Then $A$ is a positive definite, selfadjoint, unbounded, linear operator on $\dot{H}$ with compact inverse. When extended to $H$ as $A v=A P v$ it has an orthonormal eigenbasis $\left\{\varphi_{j}\right\}_{j=0}^{\infty}$ with corresponding eigenvalues $\left\{\lambda_{j}\right\}_{j=0}^{\infty}$ such that

$$
0=\lambda_{0}<\lambda_{1} \leq \lambda_{2} \leq \cdots \leq \lambda_{j} \leq \cdots, \quad \lambda_{j} \rightarrow \infty .
$$

The first eigenfunction is constant, $\varphi_{0}=|\mathcal{D}|^{-\frac{1}{2}}$.

We define seminorms and norms

$$
\begin{gathered}
|v|_{\alpha}=\left(\sum_{j=1}^{\infty} \lambda_{j}^{\alpha}\left|\left\langle v, \varphi_{j}\right\rangle\right|^{2}\right)^{\frac{1}{2}}, \quad \alpha \in \mathbf{R}, \\
\|v\|_{\alpha}=\left(|v|_{\alpha}^{2}+\left|\left\langle v, \varphi_{0}\right\rangle\right|^{2}\right)^{\frac{1}{2}}, \quad \alpha \in \mathbf{R},
\end{gathered}
$$

and corresponding spaces

$$
\dot{H}^{\alpha}=D\left(A^{\frac{\alpha}{2}}\right)=\left\{v \in \dot{H}:|v|_{\alpha}<\infty\right\}, \quad H^{\alpha}=\left\{v \in H:\|v\|_{\alpha}<\infty\right\} .
$$

For integer order $\alpha=k \geq 0, H^{k}$ coincides with the standard Sobolev spaces with $\|\cdot\|_{k}$ equivalent to the standard norm $\|\cdot\|_{H^{k}}$. For example,

$$
\|v\|_{1}^{2}=|v|_{1}^{2}+\left|\left\langle v, \varphi_{0}\right\rangle\right|^{2}=\|\nabla v\|^{2}+\left|\left\langle v, \varphi_{0}\right\rangle\right|^{2}
$$

is equivalent to the standard norm $\|v\|_{H^{1}}^{2}$ by the Poincaré inequality.

2.2. The semigroup. The operator $-A^{2}$ is the infinitesimal generator of an analytic semigroup $\mathrm{e}^{-t A^{2}}$ on $H$,

$$
\begin{aligned}
\mathrm{e}^{-t A^{2}} v & =\sum_{j=0}^{\infty} \mathrm{e}^{-t \lambda_{j}^{2}}\left\langle v, \varphi_{j}\right\rangle \varphi_{j}=\sum_{j=1}^{\infty} \mathrm{e}^{-t \lambda_{j}^{2}}\left\langle v, \varphi_{j}\right\rangle \varphi_{j}+\left\langle v, \varphi_{0}\right\rangle \varphi_{0} \\
& =\mathrm{e}^{-t A^{2}} P v+(I-P) v .
\end{aligned}
$$

The analyticity implies that

$$
\left\|A^{\alpha} \mathrm{e}^{-t A^{2}} v\right\| \leq C t^{-\frac{\alpha}{2}} \mathrm{e}^{-c t}\|v\|, \quad v \in H, \alpha>0 .
$$

2.3. The finite element method. Let $\left\{\mathcal{T}_{h}\right\}_{h>0}$ denote a family of regular triangulations of $\mathcal{D}$ with maximal mesh size $h$. Let $S_{h}$ be the space of continuous functions on $\mathcal{D}$, which are piecewise polynomials of degree $\leq 1$ with respect to $\mathcal{T}_{h}$. Hence, $S_{h} \subset H^{1}$. We also define $\dot{S}_{h}=P S_{h}$; that is,

$$
\dot{S}_{h}=\left\{v_{h} \in S_{h}: \int_{\mathcal{D}} v_{h} \mathrm{~d} x=0\right\} .
$$

The space $\dot{S}_{h}$ is introduced only for the purpose of theory but not for computation. Now we define the "discrete Laplacian" $A_{h}: S_{h} \rightarrow \dot{S}_{h}$ by

$$
\left\langle A_{h} v_{h}, w_{h}\right\rangle=\left\langle\nabla v_{h}, \nabla w_{h}\right\rangle, \quad \forall v_{h} \in S_{h}, w_{h} \in \dot{S}_{h} .
$$

We note that

$$
\left|v_{h}\right|_{1}=\left\|A^{\frac{1}{2}} v_{h}\right\|=\left\|\nabla v_{h}\right\|=\left\|A_{h}^{\frac{1}{2}} v_{h}\right\|, \quad v_{h} \in S_{h} .
$$


The operator $A_{h}$ is selfadjoint, positive definite on $\dot{S}_{h}$, positive semidefinite on $S_{h}$, and $A_{h}$ has an orthonormal eigenbasis $\left\{\varphi_{h, j}\right\}_{j=0}^{N_{h}}$ with corresponding eigenvalues $\left\{\lambda_{h, j}\right\}_{j=0}^{N_{h}}$. We have

$$
0=\lambda_{h, 0}<\lambda_{h, 1} \leq \cdots \leq \lambda_{h, j} \leq \cdots \leq \lambda_{h, N_{h}},
$$

and $\varphi_{h, 0}=\varphi_{0}=|\mathcal{D}|^{-\frac{1}{2}}$. Moreover, we define $\mathrm{e}^{-t A_{h}^{2}}: S_{h} \rightarrow S_{h}$ by

$$
\mathrm{e}^{-t A_{h}^{2}} v_{h}=\sum_{j=0}^{N_{h}} \mathrm{e}^{-t \lambda_{h, j}}\left\langle v_{h}, \varphi_{h, j}\right\rangle \varphi_{h, j}=\sum_{j=1}^{N_{h}} \mathrm{e}^{-t \lambda_{h, j}}\left\langle v_{h}, \varphi_{h, j}\right\rangle \varphi_{h, j}+\left\langle v_{h}, \varphi_{0}\right\rangle \varphi_{0},
$$

and the orthogonal projector $P_{h}: H \rightarrow S_{h}$ by

$$
\left\langle P_{h} v, w_{h}\right\rangle=\left\langle v, w_{h}\right\rangle \quad \forall v \in H, w_{h} \in S_{h} .
$$

Clearly, $P_{h}: \dot{H} \rightarrow \dot{S}_{h}$ and

$$
\mathrm{e}^{-t A_{h}^{2}} P_{h} v=\mathrm{e}^{-t A_{h}^{2}} P_{h} P v+(I-P) v .
$$

We have a discrete analog of 2.5,

$$
\left\|A_{h}^{\alpha} \mathrm{e}^{-t A_{h}^{2}} v_{h}\right\| \leq C t^{-\frac{\alpha}{2}} \mathrm{e}^{-c t}\left\|v_{h}\right\|, \quad v_{h} \in S_{h}, \alpha>0 .
$$

Finally, we define the Ritz projector $R_{h}: \dot{H}^{1} \rightarrow \dot{S}_{h}$ by

$$
\left\langle\nabla R_{h} v, \nabla w_{h}\right\rangle=\left\langle\nabla v, \nabla w_{h}\right\rangle, \quad \forall v \in \dot{H}^{1}, w_{h} \in \dot{S}_{h} .
$$

We extend it to $R_{h}: H^{1} \rightarrow S_{h}$ by

$$
R_{h} v=R_{h} P v+(I-P) v, \quad v \in H^{1} .
$$

We then have the following bound for $R_{h} v-v=\left(R_{h}-I\right) P v$ (cf. [13, Ch. 1])

$$
\left\|R_{h} v-v\right\| \leq C h^{\beta}|v|_{\beta}, \quad v \in H^{\beta}, \beta \in[1,2] .
$$

In order to simplify the presentation, we assume that $P_{h}$ is bounded with respect to the $H^{1}$ and $L_{4}$ norms, and that we have an inverse bound for $A_{h}$,

$$
\begin{array}{ll}
\left\|P_{h} v\right\|_{1} \leq C\|v\|_{1}, & v \in H^{1}, \\
\left\|P_{h} v\right\|_{L_{4}} \leq C\|v\|_{L_{4}}, & v \in L_{4}(\mathcal{D}), \\
\left\|A_{h} v_{h}\right\| \leq C h^{-2}\left\|v_{h}\right\|, & v_{h} \in S_{h} .
\end{array}
$$

This holds, for example, if the mesh family $\left\{\mathcal{T}_{h}\right\}_{h>0}$ is quasi-uniform; that is, the area of $\tau \in \mathcal{T}_{h}$ is bounded below by $c h^{d}$, with $c>0$ independent of $h$.

2.4. The Wiener process. We recall the definitions of the trace and the HilbertSchmidt norm of a linear operator $T$ on $H$ :

$$
\operatorname{Tr}(T)=\sum_{k=1}^{\infty}\left\langle T f_{k}, f_{k}\right\rangle, \quad\|T\|_{\mathrm{HS}}=\left(\sum_{k=1}^{\infty}\left\|T f_{k}\right\|^{2}\right)^{\frac{1}{2}}
$$

where $\left\{f_{k}\right\}_{k=1}^{\infty}$ is an arbitrary orthonormal basis of $H$. 
Let $(\Omega, \mathcal{F}, \mathbf{P})$ be a probability space. Let $Q$ be a selfadjoint, positive semidefinite, bounded, linear operator on $H$ with $\operatorname{Tr}(Q)<\infty$. Let $\left\{e_{k}\right\}_{k=1}^{\infty}$ be an orthonormal eigenbasis for $Q$ with eigenvalues $\left\{\gamma_{k}\right\}_{k=1}^{\infty}$. Then we define the $Q$-Wiener process

$$
W(t)=\sum_{k=1}^{\infty} \gamma_{k}^{\frac{1}{2}} \beta_{k}(t) e_{k}
$$

where the $\beta_{k}$ are real-valued, independent Brownian motions. The series converges in $L_{2}(\Omega, H)$; that is, with respect to the norm $\|v\|_{L_{2}(\Omega, H)}=\left(\mathbf{E}\left[\|v\|^{2}\right]\right)^{\frac{1}{2}}$. The process $W$ generates a filtration $\left\{\mathcal{F}_{t}\right\}_{t \geq 0}$ so that it becomes a square integrable martingale and so that we can integrate with respect to $W$. In the sequel we work in the resulting filtered probabality space $\left(\Omega, \mathcal{F}, \mathbf{P},\left\{\mathcal{F}_{t}\right\}_{t \geq 0}\right)$. We refer to [6] for the details. The $Q$-Wiener process can be defined also when the covariance operator has infinite trace but this is not needed in the present work.

2.5. The stochastic convolution. We now define (cf. [6])

$$
W_{A}(t)=\int_{0}^{t} \mathrm{e}^{-(t-s) A^{2}} \mathrm{~d} W(s)=\int_{0}^{t} \mathrm{e}^{-(t-s) A^{2}} P \mathrm{~d} W(s)+(I-P) W(t),
$$

where 2.4 was also used, and similarly, by 2.8,

$$
\begin{aligned}
W_{A_{h}}(t) & =\int_{0}^{t} \mathrm{e}^{-(t-s) A_{h}^{2}} P_{h} \mathrm{~d} W(s) \\
& =\int_{0}^{t} \mathrm{e}^{-(t-s) A_{h}^{2}} P_{h} P \mathrm{~d} W(s)+(I-P) W(t) .
\end{aligned}
$$

Hence, the constant eigenmodes cancel:

$$
W_{A_{h}}(t)-W_{A}(t)=\int_{0}^{t}\left(\mathrm{e}^{-(t-s) A_{h}^{2}} P_{h}-\mathrm{e}^{-(t-s) A^{2}}\right) P \mathrm{~d} W(s)
$$

These convolutions were studied in [12. We quote the following results from there. We use the norms

$$
\|v\|_{L_{2}\left(\Omega, \dot{H}^{\beta}\right)}=\left(\mathbf{E}\left[|v|_{\beta}^{2}\right]\right)^{\frac{1}{2}}
$$

THEOREM 2.1. If $\left\|A^{\frac{\beta-2}{2}} Q^{\frac{1}{2}}\right\|_{\mathrm{HS}}<\infty$ for some $\beta \geq 2$, then

$$
\left\|W_{A}(t)\right\|_{L_{2}\left(\Omega, \dot{H}^{\beta}\right)} \leq C\left\|A^{\frac{\beta-2}{2}} Q^{\frac{1}{2}}\right\|_{\mathrm{HS}}, \quad t \geq 0
$$

TheOrem 2.2. If $\left\|Q^{\frac{1}{2}}\right\|_{\mathrm{HS}}<\infty$, then

$$
\left\|W_{A_{h}}(t)-W_{A}(t)\right\|_{L_{2}(\Omega, H)} \leq C h^{2}|\log h|\left\|Q^{\frac{1}{2}}\right\|_{\mathrm{HS}}, \quad t \geq 0
$$

Note that $\beta=2$ in the latter theorem. In 12 these are stated with a wider range of the order $\beta$, but this is not needed in the present work. 
2.6. Gronwall's lemma. We need a generalized Gronwall lemma, see 8 , Lemma 7.1.1]. A shorter proof can found in [7.

Lemma 2.3 (Generalized Gronwall lemma). Let $\varphi \in L_{1}([0, T], \mathbf{R})$ be a nonnegative function. If

$$
\varphi(t) \leq A t^{-1+\alpha}+B \int_{0}^{t}(t-s)^{-1+\beta} \varphi(s) \mathrm{d} s, \quad t \in(0, T],
$$

with constants $A, B \geq 0$ and $\alpha, \beta>0$, then there is a constant $C=C(B, T, \alpha, \beta)$ such that

$$
\varphi(t) \leq C A t^{-1+\alpha}, \quad t \in(0, T]
$$

We also use the standard Gronwall lemma:

Lemma 2.4 (Gronwall's lemma). Let $\varphi \in L_{1}([0, T], \mathbf{R})$. If

$$
\varphi(t) \leq A+C t+B \int_{0}^{t} \varphi(s) \mathrm{d} s, \quad t \in[0, T]
$$

for some constants $A, C \geq 0$ and $B>0$, then

$$
\varphi(t) \leq\left(A+\frac{C}{B}\right) \mathrm{e}^{B t}, \quad t \in[0, T]
$$

2.7. Bounds for the nonlinear term. Recall that the standard Sobolev norm $\|\cdot\|_{H^{k}}$ is equivalent to the norm $\|\cdot\|_{k}$ in $(2.2)$ for integer $k \geq 0$. Most of the following estimates are well known and the detailed proofs are just included for pedagogical reasons, for example, to clarify the role of the projector $P$ and the difference between the standard Sobolev norm $\|\cdot\|_{H^{k}}$ and the "abstract" norm $\|\cdot\|_{k}$.

Lemma 2.5. For $u, v \in H^{3}$ and $f(s)=s^{3}-s$ we have

$$
\begin{aligned}
\|\Delta f(u)\| & \leq C\left(1+\|u\|_{1}^{2}\right)\|u\|_{3}, \\
\left\|A_{h}^{-\frac{1}{2}} P(f(u)-f(v))\right\| & \leq C\left(1+\|u\|_{1}^{2}+\|v\|_{1}^{2}\right)\|u-v\| .
\end{aligned}
$$

Proof. We have $f^{\prime}(s)=3 s^{2}-s, f^{\prime \prime}(s)=6 s$. Using Hölder's inequality, Sobolev's inequality $\|u\|_{L_{6}} \leq C\|u\|_{H^{1}}$ (for $\left.d \leq 3\right)$, and $\|u\|_{H^{k}} \leq C\|u\|_{k}$, we get

$$
\begin{aligned}
\|\Delta f(u)\| & =\left\|f^{\prime}(u) \Delta u+f^{\prime \prime}(u)|\nabla u|^{2}\right\| \\
& \leq\left\|f^{\prime}(u)\right\|_{L_{3}}\|\Delta u\|_{L_{6}}+\left\|f^{\prime \prime}(u)\right\|_{L_{6}}\|\nabla u\|_{L_{6}}^{2} \\
& \leq C\left(1+\|u\|_{L_{6}}^{2}\right)\|\Delta u\|_{L_{6}}+C\|u\|_{L_{6}}\|\nabla u\|_{L_{6}}^{2} \\
& \leq C\left(1+\|u\|_{H^{1}}^{2}\right)\|u\|_{H^{3}}+C\|u\|_{H^{1}}\|u\|_{H^{2}}^{2} \\
& \leq C\left(1+\|u\|_{1}^{2}\right)\|u\|_{3}+C\|u\|_{1}\|u\|_{2}^{2} \\
& \leq C\left(1+\|u\|_{1}^{2}\right)\|u\|_{3},
\end{aligned}
$$

where we used $\|u\|_{2} \leq C\|u\|_{1}^{\frac{1}{2}}\|u\|_{3}^{\frac{1}{2}}$ in the last step. This proves 2.17). 
For (2.18) we apply (2.6) and the Hölder and Sobolev inequalities $(d \leq 3)$ to get

$$
\begin{aligned}
\left\|A_{h}^{-\frac{1}{2}} P \varphi\right\| & =\sup _{v_{h} \in S_{h}} \frac{\left\langle A_{h}^{-\frac{1}{2}} P \varphi, v_{h}\right\rangle}{\left\|v_{h}\right\|}=\sup _{v_{h} \in S_{h}} \frac{\left\langle\varphi, A_{h}^{-\frac{1}{2}} P v_{h}\right\rangle}{\left\|v_{h}\right\|} \\
& =\sup _{w_{h} \in \dot{S}_{h}} \frac{\left\langle\varphi, w_{h}\right\rangle}{\left|w_{h}\right|_{1}} \leq \sup _{w_{h} \in \dot{S}_{h}} \frac{\|\varphi\|_{L_{6 / 5}}\left\|w_{h}\right\|_{L_{6}}}{\left|w_{h}\right|_{1}} \leq C\|\varphi\|_{L_{6 / 5}} .
\end{aligned}
$$

We use this with $\varphi=f(u)-f(v)=\int_{0}^{1} f^{\prime}\left(u_{s}\right) \mathrm{d} s(u-v)$, where $u_{s}=s u+(1-s) v$, and Hölder's and Sobolev's inequalities to get

$$
\begin{aligned}
& \| A_{h}^{-\frac{1}{2}} P(f(u)-f(v))\|=\| A_{h}^{-\frac{1}{2}} P \varphi\|\leq C\| \varphi \|_{L_{6 / 5}} \\
& \quad \leq C \int_{0}^{1}\left\|f^{\prime}\left(u_{s}\right)\right\|_{L_{3}} \mathrm{~d} s\|u-v\| \leq C \int_{0}^{1}\left(1+\left\|u_{s}\right\|_{L_{6}}^{2}\right) \mathrm{d} s\|u-v\| \\
& \quad \leq C \int_{0}^{1}\left(1+\left\|u_{s}\right\|_{1}^{2}\right) \mathrm{d} s\|u-v\| \leq C\left(1+\|u\|_{1}^{2}+\|v\|_{1}^{2}\right)\|u-v\| .
\end{aligned}
$$

This is 2.18).

\section{The Cahn-Hilliard-Cook equation.}

3.1. The continuous problem. The Cahn-Hilliard-Cook equation is

$$
\begin{array}{ll}
\mathrm{d} u-\Delta w \mathrm{~d} t=\mathrm{d} W & \text { in } \mathcal{D} \times(0, T], \\
w=-\Delta u+f(u) & \text { in } \mathcal{D} \times(0, T], \\
\frac{\partial u}{\partial n}=\frac{\partial w}{\partial n}=0 & \text { on } \partial \mathcal{D} \times(0, T], \\
u(0)=u_{0} & \text { in } \mathcal{D} .
\end{array}
$$

The finite element approximation is based on its weak form, which is (formally)

$$
\begin{array}{ll}
\langle u(t), v\rangle-\left\langle u_{0}, v\right\rangle+\int_{0}^{t}\langle\nabla w(s), \nabla v\rangle \mathrm{d} s=\langle W(t), v\rangle, & t \in(0, T], \\
\langle w, v\rangle=\langle\nabla u, \nabla v\rangle+\langle f(u), v\rangle, & t \in(0, T],
\end{array}
$$

for all $v \in \dot{H}^{1}$. With the operator $A$, defined in $\S 2.1$, we write 3.1 in the formal abstract form on $H=L_{2}(\mathcal{D})$ :

$$
\mathrm{d} X+\left(A^{2} X+A f(X)\right) \mathrm{d} t=\mathrm{d} W, \quad t \in(0, T] ; \quad X(0)=X_{0} .
$$

A weak solution of 3.3 is an adapted $H$-valued process $X$, which is continuous almost surely and satisfies the equation

$$
\langle X(t), v\rangle-\left\langle X_{0}, v\right\rangle+\int_{0}^{t}\left(\left\langle X(s), A^{2} v\right\rangle+\langle f(X(s)), A v\rangle\right) \mathrm{d} s=\langle W(t), v\rangle
$$

almost surely for all $v \in \dot{H}^{4}=D\left(A^{2}\right), t \in[0, T]$, where we also require the integrand in the deterministic integral to be in $L_{1}([0, T], \mathbf{R})$ almost surely. A mild solution of (3.3) is an adapted $H$-valued process $X$, continuous almost surely, which satisfies

$$
X(t)=\mathrm{e}^{-t A^{2}} X_{0}-\int_{0}^{t} A \mathrm{e}^{-(t-s) A^{2}} f(X(s)) \mathrm{d} s+\int_{0}^{t} \mathrm{e}^{-(t-s) A^{2}} \mathrm{~d} W(s),
$$

almost surely for $t \in[0, T]$, where we also require that the first integrand is in $L_{1}([0, T], H)$ and the stochastic integral exists almost surely. 
3.2. The finite element problem. Recalling 3.2 , we define the finite element solution $u_{h}(t), w_{h}(t) \in S_{h}$ of (3.1) by

$$
\begin{array}{ll}
\left\langle u_{h}(t), v_{h}\right\rangle-\left\langle u_{0}, v_{h}\right\rangle+\int_{0}^{t}\left\langle\nabla w_{h}(s), \nabla v_{h}\right\rangle \mathrm{d} s=\left\langle W(t), v_{h}\right\rangle, & t \in(0, T], \\
\left\langle w_{h}, v_{h}\right\rangle=\left\langle\nabla u_{h}, \nabla v_{h}\right\rangle+\left\langle f\left(u_{h}\right), v_{h}\right\rangle, & t \in(0, T],
\end{array}
$$

for all $v_{h} \in S_{h}$. With the operators $A_{h}, P_{h}$ from $\S 2.3$ we write this as an abstract equation in $S_{h}$ :

$$
\mathrm{d} X_{h}+\left(A_{h}^{2} X_{h}+A_{h} P_{h} f\left(X_{h}\right)\right) \mathrm{d} t=P_{h} \mathrm{~d} W, \quad t \in(0, T] ; \quad X_{h}(0)=P_{h} X_{0} .
$$

Since $S_{h}$ is finite-dimensional and $f$ is a polynomial, it is easy to see using standard arguments that (3.6) has a unique solution $X_{h}$, adapted, continuous almost surely, satisfying both

$$
X_{h}(t)-P_{h} X_{0}+\int_{0}^{t}\left(A_{h}^{2} X_{h}(s)+A_{h} P_{h} f\left(X_{h}(s)\right)\right) \mathrm{d} s=P_{h} W(t),
$$

and

$$
X_{h}(t)=\mathrm{e}^{-t A_{h}^{2}} P_{h} X_{0}-\int_{0}^{t} \mathrm{e}^{-(t-s) A_{h}^{2}} A_{h} P_{h} f\left(X_{h}(s)\right) \mathrm{d} s+\int_{0}^{t} \mathrm{e}^{-(t-s) A_{h}^{2}} P_{h} \mathrm{~d} W(s),
$$

almost surely for $t \in[0, T]$.

3.3. A Lyapunov functional. The deterministic Cahn-Hilliard equation defines a gradient flow in $\dot{H}^{-1}$ for the energy functional

$$
J(u)=\frac{1}{2}\|\nabla u\|^{2}+\int_{\mathcal{D}} F(u) \mathrm{d} x, \quad u \in H^{1},
$$

where $F(s)=\frac{1}{4} s^{4}-\frac{1}{2} s^{2}$ is a primitive of $f(s)=s^{3}-s$. This is a Lyapunov functional for the deterministic Cahn-Hilliard equation, which implies that $J(X(t))$ does not increase along solution paths. For the stochastic equation this is not true, but we have a bound for the expected value of $J(X(t))$.

TheOrem 3.1. Assume that $\left\|A^{\frac{1}{2}} Q^{\frac{1}{2}}\right\|_{\mathrm{HS}}<\infty$ and that $X_{0}$ is $\mathcal{F}_{0}$-measurable with values in $H^{1}$ satisfying $\mathbf{E}\left[J\left(X_{0}\right)\right]<\infty$. If $X$ is a weak solution of (3.3) and $X_{h}$ is the solution of (3.6), then, for all $t>0$, we have

$$
\mathbf{E}[J(X(t))]+\mathbf{E}\left[\int_{0}^{t}\left|J^{\prime}(X(s))\right|_{1}^{2} \mathrm{~d} s\right] \leq C\left(\mathbf{E}\left[J\left(X_{0}\right)\right]+K_{Q} t+K_{Q}^{2} t^{2}\right)
$$

and

$$
\mathbf{E}\left[J\left(X_{h}(t)\right)\right]+\mathbf{E}\left[\int_{0}^{t}\left|J^{\prime}\left(X_{h}(s)\right)\right|_{1}^{2} \mathrm{~d} s\right] \leq C\left(\mathbf{E}\left[J\left(P_{h} X_{0}\right)\right]+K_{Q} t+K_{Q}^{2} t^{2}\right),
$$

where $K_{Q}=\left\|A^{\frac{1}{2}} Q^{\frac{1}{2}}\right\|_{\mathrm{HS}}^{2}+\left\|Q^{\frac{1}{2}}\right\|_{\mathrm{HS}}^{2}$.

Proof. We prove (3.9); the proof of (3.8) is obtained in a similar way by approximating (3.3) by Galerkin's method based on the eigenbasis of $A$ instead of the finite element Galerkin method used in (3.6) (see also [5).

We consider (3.6) as an Itô differential equation in $S_{h}$ driven by $P_{h} W$, which is a $Q_{h}$-Wiener process in $S_{h}$ with $Q_{h}=P_{h} Q P_{h}$. By assumption 2.12 it follows that 
$\mathbf{E}\left[J\left(P_{h} X_{0}\right)\right]<\infty$, if $\mathbf{E}\left[J\left(X_{0}\right)\right]<\infty$. By applying Itô's formula ([, Theorem 4.17]) to $J\left(X_{h}(t)\right)$, we obtain

$$
\begin{aligned}
J\left(X_{h}(t)\right)= & J\left(X_{h}(0)\right)+\int_{0}^{t}\left\langle J^{\prime}\left(X_{h}(s)\right), \mathrm{d} X_{h}(s)\right\rangle+\frac{1}{2} \int_{0}^{t} \operatorname{Tr}\left(J^{\prime \prime}\left(X_{h}(s)\right) Q_{h}\right) \mathrm{d} s \\
= & J\left(P_{h} X_{0}\right)+\int_{0}^{t}\left\langle J^{\prime}\left(X_{h}(s)\right),-A_{h}^{2} X_{h}(s)-A_{h} P_{h} f\left(X_{h}(s)\right)\right\rangle \mathrm{d} s \\
& +\int_{0}^{t}\left\langle J^{\prime}\left(X_{h}(s)\right), P_{h} \mathrm{~d} W(s)\right\rangle+\frac{1}{2} \int_{0}^{t} \operatorname{Tr}\left(J^{\prime \prime}\left(X_{h}(s)\right) Q_{h}\right) \mathrm{d} s .
\end{aligned}
$$

With a slight abuse of notation we consider here $J$ as a function $S_{h} \rightarrow \mathbf{R}$ and we compute $J^{\prime}\left(u_{h}\right) \in S_{h}$ and $J^{\prime \prime}\left(u_{h}\right): S_{h} \rightarrow S_{h}$ as follows:

$$
\left\langle J^{\prime}\left(u_{h}\right), v_{h}\right\rangle=\left\langle\nabla u_{h}, \nabla v_{h}\right\rangle+\left\langle f\left(u_{h}\right), v_{h}\right\rangle=\left\langle A_{h} u_{h}+P_{h} f\left(u_{h}\right), v_{h}\right\rangle
$$

and

$$
\left\langle J^{\prime \prime}\left(u_{h}\right) v_{h}, w_{h}\right\rangle=\left\langle\nabla v_{h}, \nabla w_{h}\right\rangle+\left\langle f^{\prime}\left(u_{h}\right) v_{h}, w_{h}\right\rangle=\left\langle A_{h} v_{h}+P_{h}\left[f^{\prime}\left(u_{h}\right) v_{h}\right], w_{h}\right\rangle
$$

for $u_{h}, v_{h}, w_{h} \in S_{h}$, so that

$$
J^{\prime}\left(u_{h}\right)=A_{h} u_{h}+P_{h} f\left(u_{h}\right), \quad J^{\prime \prime}\left(u_{h}\right)=A_{h}+P_{h}\left[f^{\prime}\left(u_{h}\right) \cdot\right] .
$$

Hence, by (2.6),

$$
\begin{aligned}
J\left(X_{h}(t)\right)+\int_{0}^{t}\left|J^{\prime}\left(X_{h}(s)\right)\right|_{1}^{2} \mathrm{~d} s= & J\left(P_{h} X_{0}\right)+\int_{0}^{t}\left\langle J^{\prime}\left(X_{h}(s)\right), P_{h} \mathrm{~d} W(s)\right\rangle \\
& +\frac{1}{2} \int_{0}^{t} \operatorname{Tr}\left(J^{\prime \prime}\left(X_{h}(s)\right) Q_{h}\right) \mathrm{d} s .
\end{aligned}
$$

The stochastic integral is a martingale, so that $\mathbf{E}\left[\int_{0}^{t}\left\langle J^{\prime}\left(X_{h}\right), P_{h} \mathrm{~d} W\right\rangle\right]=0$, and hence

$$
\begin{aligned}
& \mathbf{E}\left[J\left(X_{h}(t)\right)\right]+\mathbf{E}\left[\int_{0}^{t}\left|J^{\prime}\left(X_{h}(s)\right)\right|_{1}^{2} \mathrm{~d} s\right] \\
& =\mathbf{E}\left[J\left(P_{h} X_{0}\right)\right]+\frac{1}{2} \mathbf{E}\left[\int_{0}^{t} \operatorname{Tr}\left(J^{\prime \prime}\left(X_{h}(s)\right) Q_{h}\right) \mathrm{d} s\right]
\end{aligned}
$$

We now compute

$$
\operatorname{Tr}\left(J^{\prime \prime}\left(X_{h}(s)\right) Q_{h}\right)=\operatorname{Tr}\left(A_{h} Q_{h}\right)+\operatorname{Tr}\left(P_{h}\left[f^{\prime}\left(X_{h}(s)\right) \cdot\right] Q_{h}\right)
$$

by the definition in 2.13. Recall that for $S, T \in \mathcal{B}(H)$ we have $\operatorname{Tr}(T S)=\operatorname{Tr}(S T)$ provided that either $S$ or $T$ has finite trace. If, in addition, the operators $S$ and $T$ are positive semidefinite as well, then $\operatorname{Tr}(S T)=\left\|S^{\frac{1}{2}} T^{\frac{1}{2}}\right\|_{\mathrm{HS}}^{2}$. Thus,

$$
\begin{aligned}
\operatorname{Tr}\left(A_{h} Q_{h}\right) & =\operatorname{Tr}\left(A_{h} P_{h} Q P_{h}\right)=\operatorname{Tr}\left(P_{h} A_{h} P_{h} Q\right)=\left\|A_{h}^{\frac{1}{2}} P_{h} Q^{\frac{1}{2}}\right\|_{\mathrm{HS}}^{2} \\
& \leq\left\|A_{h}^{\frac{1}{2}} P_{h} A^{-\frac{1}{2}}\right\|_{B(\dot{H})}^{2}\left\|A^{\frac{1}{2}} Q^{\frac{1}{2}}\right\|_{\mathrm{HS}}^{2} .
\end{aligned}
$$

Here we use 2.6 and 2.12 to get

$$
\left\|A_{h}^{\frac{1}{2}} P_{h} A^{-\frac{1}{2}} v\right\|=\left|P_{h} A^{-\frac{1}{2}} v\right|_{1} \leq C\left|A^{-\frac{1}{2}} v\right|_{1}=C\|v\|, \quad v \in \dot{H},
$$


so that $\left\|A_{h}^{\frac{1}{2}} P_{h} A^{-\frac{1}{2}}\right\|_{B(\dot{H})} \leq C$. Hence, with $K_{Q}=\left\|A^{\frac{1}{2}} Q^{\frac{1}{2}}\right\|_{\mathrm{HS}}^{2}+\left\|Q^{\frac{1}{2}}\right\|_{\mathrm{HS}}^{2}$,

$$
\operatorname{Tr}\left(A_{h} Q_{h}\right) \leq C\left\|A^{\frac{1}{2}} Q^{\frac{1}{2}}\right\|_{\mathrm{HS}}^{2} \leq C K_{Q} .
$$

Let $\left\{e_{h, k}\right\}_{k=0}^{N_{h}}$ be an orthonormal eigenbasis of $Q_{h}$ and $\left\{\gamma_{h, k}\right\}_{k=0}^{N_{h}}$ the corresponding eigenvalues. We get

$$
\begin{aligned}
\operatorname{Tr}\left(P_{h}\left[f^{\prime}\left(X_{h}\right) \cdot\right] Q_{h}\right)=\sum_{k=0}^{N_{h}}\left\langle P_{h}\left[f^{\prime}\left(X_{h}\right) Q_{h} e_{h, k}\right], e_{h, k}\right\rangle \\
=\sum_{k=0}^{N_{h}} \gamma_{h, k}\left\langle f^{\prime}\left(X_{h}\right) e_{h, k}, e_{h, k}\right\rangle=\sum_{k=0}^{N_{h}}\left\langle f^{\prime}\left(X_{h}\right) Q_{h}^{\frac{1}{2}} e_{h, k}, Q_{h}^{\frac{1}{2}} e_{h, k}\right\rangle .
\end{aligned}
$$

By using the bound $\left|f^{\prime}(s)\right| \leq C\left(1+s^{2}\right)$ and Hölder's and Sobolev's inequalities we get

$$
\left|\left\langle f^{\prime}(u) v, v\right\rangle\right| \leq C\left(1+\|u\|_{L_{4}}^{2}\right)\|v\|_{L_{4}}^{2} \leq C\left(1+\|u\|_{L_{4}}^{2}\right)\|v\|_{H^{1}}^{2} \leq C\left(1+\|u\|_{L_{4}}^{2}\right)\|v\|_{1}^{2} .
$$

By 2.3 and 2.6 we have, for $v_{h} \in S_{h}$,

$$
\left\|v_{h}\right\|_{1}^{2}=\left|v_{h}\right|_{1}^{2}+\left\langle v_{h}, \varphi_{0}\right\rangle^{2}=\left\|A_{h}^{\frac{1}{2}} v_{h}\right\|^{2}+\left\langle v_{h}, \varphi_{0}\right\rangle^{2},
$$

so that, by (3.13),

$$
\begin{aligned}
\sum_{k=0}^{N_{h}} \| & Q_{h}^{\frac{1}{2}} e_{h, k}\left\|_{1}^{2}=\sum_{k=0}^{N_{h}}\right\| A_{h}^{\frac{1}{2}} Q_{h}^{\frac{1}{2}} e_{h, k} \|^{2}+\sum_{k=0}^{N_{h}}\left\langle Q_{h}^{\frac{1}{2}} e_{h, k}, \varphi_{0}\right\rangle^{2} \\
= & \sum_{k=0}^{N_{h}} \gamma_{h, k}\left\langle A_{h} e_{h, k}, e_{h, k}\right\rangle+\sum_{k=0}^{N_{h}} \gamma_{h, k}\left\langle e_{h, k}, \varphi_{0}\right\rangle^{2} \\
\leq & \operatorname{Tr}\left(A_{h} Q_{h}\right)+\operatorname{Tr}\left(Q_{h}\right) \leq \operatorname{Tr}\left(A_{h} Q_{h}\right)+\operatorname{Tr}(Q) \\
\leq & C\left\|A^{\frac{1}{2}} Q^{\frac{1}{2}}\right\|_{\mathrm{HS}}^{2}+\left\|Q^{\frac{1}{2}}\right\|_{\mathrm{HS}}^{2} \leq C K_{Q} .
\end{aligned}
$$

Returning to (3.14), we now have

$$
\left|\operatorname{Tr}\left(P_{h}\left[f^{\prime}\left(X_{h}\right) \cdot\right] Q_{h}\right)\right| \leq C\left(1+\left\|X_{h}\right\|_{L_{4}}^{2}\right) \sum_{k=0}^{N_{h}}\left\|Q_{h}^{\frac{1}{2}} e_{h, k}\right\|_{1}^{2} \leq C\left(1+\left\|X_{h}\right\|_{L_{4}}^{2}\right) K_{Q},
$$

Using also 3.13 we conclude

$$
\left|\operatorname{Tr}\left(J^{\prime \prime}\left(X_{h}\right) Q_{h}\right)\right| \leq C K_{Q}\left(1+\left\|X_{h}\right\|_{L_{4}}^{2}\right) .
$$

It remains to relate $\left\|X_{h}\right\|_{L_{4}}$ to $J\left(X_{h}\right)$. By definition of the Lyapunov functional (3.7) and noting that $F(s)=\frac{1}{4} s^{4}-\frac{1}{2} s^{2} \geq c_{1} s^{4}-c_{2}$, we get (with new constants)

$$
J(u) \geq \frac{1}{2}\|\nabla u\|^{2}+C_{1}\|u\|_{L_{4}}^{4}-C_{2},
$$

which implies

$$
\|\nabla u\|^{2}+\|u\|_{L_{4}}^{4} \leq C_{3}(1+J(u)) .
$$


Hence, in view of 3.15,

$$
\left|\operatorname{Tr}\left(J^{\prime \prime}\left(X_{h}\right) Q_{h}\right)\right| \leq C K_{Q}\left(1+J\left(X_{h}\right)^{\frac{1}{2}}\right) .
$$

Inserting this into 3.12 gives

$$
\begin{aligned}
& \mathbf{E}\left[J\left(X_{h}(t)\right)\right]+\mathbf{E}\left[\int_{0}^{t}\left|J^{\prime}\left(X_{h}(s)\right)\right|_{1}^{2} \mathrm{~d} s\right] \\
& \quad \leq \mathbf{E}\left[J\left(P_{h} X_{0}\right)\right]+C K_{Q}\left(t+\int_{0}^{t} \mathbf{E}\left[J\left(X_{h}(s)\right)^{\frac{1}{2}}\right] \mathrm{d} s\right) .
\end{aligned}
$$

Here, by Hölder's and Young's inequalities, we have, for $\epsilon>0$,

$$
\begin{aligned}
C K_{Q} \int_{0}^{t} \mathbf{E}\left[J\left(X_{h}(s)\right)^{\frac{1}{2}}\right] \mathrm{d} s & \leq C K_{Q} t^{\frac{1}{2}}\left(\int_{0}^{t} \mathbf{E}\left[J\left(X_{h}(s)\right)\right] \mathrm{d} s\right)^{\frac{1}{2}} \\
& \leq \epsilon \int_{0}^{t} \mathbf{E}\left[J\left(X_{h}(s)\right)\right] \mathrm{d} s+C \epsilon^{-1} t K_{Q}^{2}
\end{aligned}
$$

Putting this in 3.18 gives

$$
\begin{aligned}
& \mathbf{E}\left[J\left(X_{h}(t)\right)\right]+\mathbf{E}\left[\int_{0}^{t}\left|J^{\prime}\left(X_{h}(s)\right)\right|_{1}^{2} \mathrm{~d} s\right] \\
& \quad \leq \mathbf{E}\left[J\left(P_{h} X_{0}\right)\right]+C\left(K_{Q}+\epsilon^{-1} K_{Q}^{2}\right) t+\epsilon \int_{0}^{t} \mathbf{E}\left[J\left(X_{h}(s)\right)\right] \mathrm{d} s .
\end{aligned}
$$

We apply the Gronwall Lemma 2.4 to get,

$$
\begin{gathered}
\mathbf{E}\left[J\left(X_{h}(t)\right)\right]+\mathbf{E}\left[\int_{0}^{t}\left|J^{\prime}\left(X_{h}(s)\right)\right|_{1}^{2} \mathrm{~d} s\right] \\
\leq \mathrm{e}^{\epsilon t}\left(\mathbf{E}\left[J\left(P_{h} X_{0}\right)\right]+C\left(\epsilon^{-1} K_{Q}+\epsilon^{-2} K_{Q}^{2}\right)\right) \\
\quad \leq \mathrm{e}\left(\mathbf{E}\left[J\left(P_{h} X_{0}\right)\right]+C\left(t K_{Q}+t^{2} K_{Q}^{2}\right)\right),
\end{gathered}
$$

where for each fixed $t$ we have chosen $\epsilon=t^{-1}$ to get an optimal bound.

This theorem is adapted from [5]. We have improved it in several ways. First the growth of the bound is reduced from exponential to quadratic with respect to $t$. Most importantly, we have removed the assumption that $A$ and $Q$ have a common eigenbasis and that the eigenbasis satisfies $\left\|e_{k}\right\|_{L_{\infty}} \leq C$ for all $k$. This is important because even if $A$ and $Q$ commute, this will not be true for $A_{h}$ and $Q_{h}$. This is crucial for the proof of the bound for $X_{h}$.

Note that $\left\|A^{\frac{1}{2}} Q^{\frac{1}{2}}\right\|_{\mathrm{HS}}<\infty$ implies $K_{Q}=\left\|A^{\frac{1}{2}} Q^{\frac{1}{2}}\right\|_{\mathrm{HS}}^{2}+\left\|Q^{\frac{1}{2}}\right\|_{\mathrm{HS}}^{2}<\infty$. This is 
because of the boundedness of $A^{-\frac{1}{2}}$ and

$$
\begin{aligned}
\left\|Q^{\frac{1}{2}}\right\|_{\mathrm{HS}}^{2} & =\sum_{j=1}^{\infty}\left\langle Q \varphi_{j}, \varphi_{j}\right\rangle+\left\langle Q \varphi_{0}, \varphi_{0}\right\rangle=\sum_{j=1}^{\infty}\left\|P Q^{\frac{1}{2}} \varphi_{j}\right\|^{2}+\left\langle Q \varphi_{0}, \varphi_{0}\right\rangle \\
& =\sum_{j=1}^{\infty}\left\|A^{-\frac{1}{2}} A^{\frac{1}{2}} P Q^{\frac{1}{2}} \varphi_{j}\right\|^{2}+\left\langle Q \varphi_{0}, \varphi_{0}\right\rangle \\
& \leq C \sum_{j=1}^{\infty}\left\|A^{\frac{1}{2}} P Q^{\frac{1}{2}} \varphi_{j}\right\|^{2}+\left\langle Q \varphi_{0}, \varphi_{0}\right\rangle \\
& \leq C\left\|A^{\frac{1}{2}} P Q^{\frac{1}{2}}\right\|_{\mathrm{HS}}^{2}+\left\langle Q \varphi_{0}, \varphi_{0}\right\rangle=C\left\|A^{\frac{1}{2}} Q^{\frac{1}{2}}\right\|_{\mathrm{HS}}^{2}+\left\langle Q \varphi_{0}, \varphi_{0}\right\rangle<\infty .
\end{aligned}
$$

This condition is therefore the same as the condition for regularity of order $\beta=3$ for $W_{A}(t)$ in Theorem 2.1.

We now use the previous theorem together with Chebyshev's inequality to obtain pathwise norm bounds uniformly on subsets of $\Omega$ with probability arbitrarily close to 1. In order to achieve this we first replace the bound of $\sup _{s \in[0, t]} \mathbf{E}[J(X(s))]$ from Theorem 3.1 by a bound for $\mathbf{E}\left[\sup _{s \in[0, t]}\left(\|\nabla X(s)\|^{2}+\|X(s)\|_{L_{4}}^{4}\right)\right]$.

Corollary 3.2. Assume that $\left\|A^{\frac{\gamma}{2}} Q^{\frac{1}{2}}\right\|_{\mathrm{HS}}<\infty$ for some $\gamma>1$ and that $X_{0}$ is $\mathcal{F}_{0}$-measurable with values in $H^{1}$ satisfying

$$
\left\|X_{0}\right\|_{L_{2}\left(\Omega, H^{1}\right)}^{2}+\left\|X_{0}\right\|_{L_{4}\left(\Omega, L_{4}\right)}^{4} \leq \rho
$$

for some $\rho \geq 0$. If $X$ is a weak solution of (3.3) and $X_{h}$ is the solution of (3.6), then, for $T \geq 0$,

$$
\begin{array}{r}
\mathbf{E}\left[\sup _{t \in[0, T]}\left(\|\nabla X(t)\|^{2}+\|X(t)\|_{L_{4}}^{4}\right)\right] \leq K_{T}, \\
\mathbf{E}\left[\sup _{t \in[0, T]}\left(\left\|\nabla X_{h}(t)\right\|^{2}+\left\|X_{h}(t)\right\|_{L_{4}}^{4}\right)\right] \leq K_{T},
\end{array}
$$

where $K_{T}$ depends on $\rho, K_{Q}, T$. Moreover, for every $\epsilon \in(0,1)$, there is $\Omega_{\epsilon} \subset \Omega$ with $\mathbf{P}\left(\Omega_{\epsilon}\right) \geq 1-\epsilon$ and

$$
\begin{aligned}
\|\nabla X(t)\|^{2}+\|X(t)\|_{L_{4}}^{4} \leq \epsilon^{-1} K_{T} & \text { on } \Omega_{\epsilon}, t \in[0, T], \\
\left\|\nabla X_{h}(t)\right\|^{2}+\left\|X_{h}(t)\right\|_{L_{4}}^{4} \leq \epsilon^{-1} K_{T} & \text { on } \Omega_{\epsilon}, t \in[0, T], \\
\|X(t)\|_{1}^{2}+\left\|X_{h}(t)\right\|_{1}^{2} \leq \epsilon^{-1} K_{T} & \text { on } \Omega_{\epsilon}, t \in[0, T], \\
\left\|W_{A}(t)\right\|_{3}^{2} \leq \epsilon^{-1} K_{T} & \text { on } \Omega_{\epsilon}, t \in[0, T] .
\end{aligned}
$$

We remark that the stronger assumption $\gamma>1$ is only used in the proof of 3.26 .

Proof. It is enough to prove the existence of an $\Omega_{\epsilon}$ for every $\epsilon>0$ individually in (3.23) 3.26 . We prove the bounds for $X_{h}$ and $W_{A}$; the others are proved similarly.

From (3.20) and 2.12 there follows $\mathbf{E}\left[J\left(P_{h} X_{0}\right)\right] \leq C(1+\rho)$. Using also (3.16) in 3.11 , we obtain

$$
\begin{aligned}
& \mathbf{E}\left[\sup _{t \in[0, T]}\left(\left\|\nabla X_{h}(t)\right\|^{2}+\left\|X_{h}(t)\right\|_{L_{4}}^{4}\right)\right] \\
& \leq C(1+\rho)+C \mathbf{E}\left[\sup _{t \in[0, T]}\left|\int_{0}^{t}\left\langle J^{\prime}\left(X_{h}(s)\right), P_{h} \mathrm{~d} W(s)\right\rangle\right|\right] \\
&+C \mathbf{E}\left[\sup _{t \in[0, T]}\left|\int_{0}^{t} \operatorname{Tr}\left(J^{\prime \prime}\left(X_{h}(s) Q_{h}\right)\right) \mathrm{d} s\right|\right] .
\end{aligned}
$$


The stochastic integral is $\left.\int_{0}^{t}\left\langle J^{\prime}\left(X_{h}(s)\right), P_{h} \mathrm{~d} W(s)\right\rangle=\int_{0}^{t} J^{\prime} \widetilde{\left(X_{h}(s)\right.}\right) P_{h} \mathrm{~d} W(s)$, where $\left.J^{\prime} \widetilde{\left(X_{h}(s)\right.}\right): H \rightarrow \mathbf{R}$ is defined by $J^{\prime} \widetilde{\left(X_{h}(s)\right)} v=\left\langle J^{\prime}\left(X_{h}(s)\right), v\right\rangle$. This integral is a martingale. Hence, we may use Hölder's inequality, the martingale inequality ([6, Theorem 3.8]), and the Itô isometry ([․ Corollary 4.14]) to get

$$
\begin{aligned}
& \left.\left(\mathbf{E}\left[\sup _{t \in[0, T]} \mid \int_{0}^{t} J^{\prime} \widetilde{\left(X_{h}(s)\right.}\right) P_{h} \mathrm{~d} W(s) \mid\right]\right)^{2} \leq \mathbf{E}\left[\sup _{t \in[0, T]}\left|\int_{0}^{t} \widetilde{J^{\prime}\left(\widetilde{X_{h}(s)}\right)} P_{h} \mathrm{~d} W(s)\right|^{2}\right]
\end{aligned}
$$

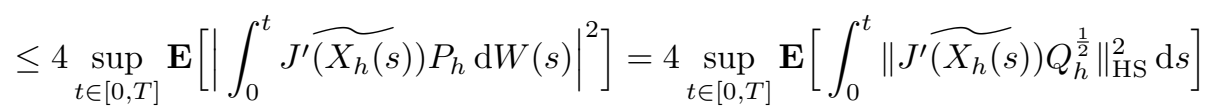

$$
\begin{aligned}
& \left.=4 \mathbf{E}\left[\int_{0}^{T} \| \widetilde{J^{\prime}} \widetilde{\left(X_{h}(s)\right.}\right) Q_{h}^{\frac{1}{2}} \|_{\mathrm{HS}}^{2} \mathrm{~d} s\right] .
\end{aligned}
$$

Here, by 2.2,

$$
\begin{aligned}
\left.\| \widetilde{J^{\prime}\left(X_{h}(s)\right.}\right) Q_{h}^{\frac{1}{2}} \|_{\mathrm{HS}}^{2} & \leq\left\|J^{\prime}\left(X_{h}(s)\right)\right\|^{2}\left\|Q_{h}^{\frac{1}{2}}\right\|_{\mathrm{HS}}^{2} \\
& \leq\left(\left|J^{\prime}\left(X_{h}(s)\right)\right|_{0}^{2}+\left\langle J^{\prime}\left(X_{h}(s)\right), \varphi_{0}\right\rangle^{2}\right) \operatorname{Tr}(Q) \\
& \leq C\left(1+\left|J^{\prime}\left(X_{h}(s)\right)\right|_{1}^{2}+J\left(X_{h}(s)\right)\right) \operatorname{Tr}(Q),
\end{aligned}
$$

where we used (3.10) and the (rough) bounds $|u|_{0} \leq|u|_{1}$ and

$$
\begin{aligned}
& \left\langle J^{\prime}\left(u_{h}\right), \varphi_{0}\right\rangle^{2}=|\mathcal{D}|^{-1}\left(\int_{\mathcal{D}}\left(A_{h} u_{h}+P_{h}\left(u_{h}^{3}-u_{h}\right)\right) \mathrm{d} x\right)^{2} \\
& \quad=|\mathcal{D}|^{-1}\left(\int_{\mathcal{D}} P_{h}\left(u_{h}^{3}-u_{h}\right) \mathrm{d} x\right)^{2} \leq C\left(1+\left\|u_{h}\right\|_{L_{4}}^{4}\right) \leq C\left(1+J\left(u_{h}\right)\right) .
\end{aligned}
$$

By using (3.9), we conclude that

$$
\begin{aligned}
& \left.\left(\mathbf{E}\left[\sup _{t \in[0, T]} \mid \int_{0}^{t} \widetilde{J^{\prime}\left(X_{h}(s)\right.}\right) P_{h} \mathrm{~d} W(s) \mid\right]\right)^{2} \\
& \quad \leq C \operatorname{Tr}(Q)\left(T+\mathbf{E}\left[\int_{0}^{T}\left|J^{\prime}\left(X_{h}(s)\right)\right|_{1}^{2} \mathrm{~d} s\right]+T \sup _{s \in[0, T]} \mathbf{E}\left[J\left(X_{h}(s)\right)\right]\right) \leq K_{T}^{2} .
\end{aligned}
$$

Next, using (3.17) and Hölder's inequality, we have

$$
\begin{gathered}
\mathbf{E}\left[\sup _{t \in[0, T]}\left|\int_{0}^{t} \operatorname{Tr}\left(J^{\prime \prime}\left(X_{h}(s) Q_{h}\right)\right) \mathrm{d} s\right|\right] \leq \mathbf{E}\left[\int_{0}^{T}\left|\operatorname{Tr}\left(J^{\prime \prime}\left(X_{h}(s) Q_{h}\right)\right)\right| \mathrm{d} s\right] \\
\quad \leq C K_{Q} \mathbf{E}\left[\int_{0}^{T}\left(1+J\left(X_{h}(s)\right)^{\frac{1}{2}}\right) \mathrm{d} s\right] \\
\quad \leq C K_{Q} T\left(1+\sup _{s \in[0, T]} \mathbf{E}\left[J\left(X_{h}(s)\right)\right]\right) \leq K_{T},
\end{gathered}
$$

which finishes the proof of 3.22 .

In order to prove $(3.24)$ we denote

$$
F_{h}=\sup _{t \in[0, T]}\left(\left\|\nabla X_{h}(t)\right\|^{2}+\left\|X_{h}(t)\right\|_{L_{4}}^{4}\right) .
$$

We apply Chebyshev's inequality and 3.22 to get, for every $\alpha>0$,

$$
\mathbf{P}\left(\left\{\omega \in \Omega: F_{h}>\alpha\right\}\right) \leq \frac{1}{\alpha} \mathbf{E}\left[F_{h}\right] \leq \frac{K_{T}}{\alpha} .
$$


We choose $\alpha=\epsilon^{-1} K_{T}$ and set $\Omega_{\epsilon}=\left\{\omega \in \Omega: F_{h} \leq \epsilon^{-1} K_{T}\right\}$. Then

$$
\mathbf{P}\left(\Omega_{\epsilon}\right)=1-\mathbf{P}\left(\left\{\omega \in \Omega: F_{h}>\alpha\right\}\right) \geq 1-\epsilon
$$

and (3.24) holds. For 3.25 we note that

$$
\|u\|_{1}^{2} \leq\|\nabla u\|^{2}+\|u\|^{2} \leq\|\nabla u\|^{2}+C\left(1+\|u\|_{L_{4}}^{4}\right)
$$

and hence 3.25 follows from (3.21) and 3.22 after an adjustment of $K_{T}$. Finally, (3.26) follows by first employing a factorization method argument as in the proof of 6. Remark 5.11] but in the $H^{3}$-norm using the analyticity of the semigroup (this is where $\gamma>1$ is needed), and then using Chebychev's inequality as above.

4. Regularity of the solution. We quote the following from [5]. There it is assumed that $A$ and $Q$ commute and that the eigenfunctions of $A$ are uniformly bounded in the sup norm but it can be verified that these are not necessary for the following result. Recall the definitions of weak and mild solutions in (3.4) and (3.5).

TheOrEm 4.1. Let $T>0$ and assume that $\operatorname{Tr}\left(A^{\delta-1} Q\right)<\infty$ for some $\delta>0$ and that $X_{0}$ is $\mathcal{F}_{0}$-measurable with values in $H$. Then there is a unique weak solution $X$ of 3.3 .

Corollary 4.2. Assume that $\left\|A^{\frac{1}{2}} Q^{\frac{1}{2}}\right\|_{\mathrm{HS}}<\infty$ and that $X_{0}$ is $\mathcal{F}_{0}$-measurable with values in $H^{1}$ satisfying $\left\|X_{0}\right\|_{L_{2}\left(\Omega, H^{1}\right)}^{2}+\left\|X_{0}\right\|_{L_{4}\left(\Omega, L_{4}\right)}^{4} \leq \rho$ for some $\rho \geq 0$. Then the weak solution $X$ of $(3.3)$ is also a mild solution.

Proof. By (3.19), the condition $\left\|A^{\frac{1}{2}} Q^{\frac{1}{2}}\right\|_{\mathrm{HS}}<\infty$ implies $\operatorname{Tr}\left(A^{\delta-1} Q\right)<\infty$ with $\delta=1$ and hence there is a unique weak solution $X$ of $(3.3)$ by Theorem 4.1. Let $\epsilon>0$ and $\Omega_{\epsilon}$ the set defined in Corollary 3.2. We first show that $X$ satisfies (3.5) on $\Omega_{\epsilon}$. By the uniqueness weak solutions of $(3.3)$, we only have to show that the right-hand side of (3.5) satisfies (3.4) on $\Omega_{\epsilon}$. Since $W_{A}$ is the unique weak solution of $\mathrm{d} Z+A^{2} Z \mathrm{~d} t=\mathrm{d} W, Z(0)=0$ (by [6, Theorem 5.4]), it is enough to show that

$$
Y(t):=\mathrm{e}^{-t A^{2}} X_{0}-\int_{0}^{t} A \mathrm{e}^{-(t-s) A^{2}} f(X(s)) \mathrm{d} s
$$

satisfies

$$
\langle Y(t), v\rangle-\left\langle X_{0}, v\right\rangle+\int_{0}^{t}\left(\left\langle Y(s), A^{2} v\right\rangle+\langle f(X(s)), A v\rangle\right) \mathrm{d} s=0 \text { on } \Omega_{\epsilon}, v \in D\left(A^{2}\right) .
$$

This follows by standard arguments (see, e.g., [1]) as, by Sobolev's inequality and Corollary 3.2 , there is $C$ depending on $\epsilon, \rho, Q$, and $T$ such that

$$
\|f(X(s))\| \leq C\left(\|X(s)\|_{L^{2}}+\|X(s)\|_{L^{6}}^{3}\right) \leq C\left(\|X(s)\|_{L^{2}}+\|X(s)\|_{H^{1}}^{3}\right) \leq C
$$

for $t \in[0, T], \omega \in \Omega_{\epsilon}$. This also shows that the integrand of the deterministic integral in $(3.5)$ is in $L_{1}([0, T], H)$ on $\Omega_{\epsilon}$ by the analyticity of the semigroup $\mathrm{e}^{-t A^{2}}$. Finally, since $\epsilon>0$ is arbitrary and $P\left(\Omega_{\epsilon}\right)>1-\epsilon$, the statement follows.

We now show that, under the stronger assumption $\left\|A^{\frac{\gamma}{2}} Q^{\frac{1}{2}}\right\|_{\mathrm{HS}}<\infty$ for some $\gamma>1$, the solution $X(t)$ is actually in $H^{3}$ almost surely. In order to do this we write, as in the previous proof, $X(t)=Y(t)+W_{A}(t)$, where we already know from Theorem 2.1 that $W_{A}(t)$ is in $H^{3}$ almost surely. The regularity of $Y$ is studied in the next theorem. Note that we saw in the proof of Corollary 4.2 that

$$
Y(t)=X(t)-W_{A}(t)=\mathrm{e}^{-t A^{2}} X_{0}-\int_{0}^{t} A \mathrm{e}^{-(t-s) A^{2}} f(X(s)) \mathrm{d} s
$$


is a weak solution of

$$
\dot{Y}+A^{2} Y+A f(X)=0, \quad t \in(0, T] ; \quad Y(0)=X_{0},
$$

almost surely.

THEOREM 4.3. Assume that $\left\|A^{\frac{\gamma}{2}} Q^{\frac{1}{2}}\right\|_{\mathrm{HS}}<\infty$ for some $\gamma>1$ and that $X_{0}$ is $\mathcal{F}_{0}$-measurable with values in $H^{3}$ satisfying $\left\|X_{0}\right\|_{L_{2}\left(\Omega, H^{1}\right)}^{2}+\left\|X_{0}\right\|_{L_{4}\left(\Omega, L_{4}\right)}^{4} \leq \rho$ for some $\rho \geq 0$. Let $T>0$ and $\epsilon \in(0,1)$ and let $\Omega_{\epsilon}$ and $K_{T}$ be as in Corollary 3.2 such that also $\left\|X_{0}\right\|_{3} \leq C$ on $\Omega_{\epsilon}$. Let $X$ be the solution from Theorem 4.1 and $Y=X-W_{A}$. Then $X, Y \in C([0, T], H) \cap L_{\infty}\left([0, T], H^{3}\right)$ almost surely, and, for each $\omega \in \Omega_{\epsilon}$,

$$
\begin{aligned}
\|Y(t)\|_{3} \leq C\left(\left\|X_{0}\right\|_{3}, \epsilon^{-1} K_{T}, T\right) & \text { on } \Omega_{\epsilon}, t \in[0, T], \\
\|X(t)\|_{3} \leq C\left(\left\|X_{0}\right\|_{3}, \epsilon^{-1} K_{T}, T\right) & \text { on } \Omega_{\epsilon}, t \in[0, T] .
\end{aligned}
$$

Proof. First note that since $X_{0}$ is $H^{3}$-valued almost surely, it is always possible to choose $\Omega_{\epsilon}$ in Corollary 3.2 such that also $\left\|X_{0}\right\|_{3} \leq C$ on $\Omega_{\epsilon}$. The continuity of $X$ is already contained in Theorem 4.1 and the continuity of $Y$ follows from the continuity of $X$ and $W_{A}$. To show that $X, Y \in L_{\infty}\left([0, T], H^{3}\right)$ almost surely it is enough to show (4.2) and (4.3) as $\epsilon>0$ is arbitrary and $P\left(\Omega_{\epsilon}\right) \geq 1-\epsilon$. Let $t \in[0, T]$ and $\omega \in \Omega_{\epsilon}$. From Corollary 3.2 we have

$$
\|X(t)\|_{1}^{2} \leq \epsilon^{-1} K_{T}, \quad\left\|W_{A}(t)\right\|_{3} \leq \epsilon^{-1} K_{T} .
$$

We take seminorms in

$$
Y(t)=\mathrm{e}^{-t A^{2}} X_{0}-\int_{0}^{t} A \mathrm{e}^{-(t-s) A^{2}} f(X(s)) \mathrm{d} s
$$

and use 2.5 to get

$$
\begin{aligned}
|Y(t)|_{3} & \leq\left|\mathrm{e}^{-t A^{2}} X_{0}\right|_{3}+\int_{0}^{t}\left|\mathrm{e}^{-(t-s) A^{2}} A f(X(s))\right|_{3} \mathrm{~d} s \\
& =\left\|\mathrm{e}^{-t A^{2}} A^{\frac{3}{2}} X_{0}\right\|+\int_{0}^{t}\left\|A^{\frac{3}{2}} \mathrm{e}^{-(t-s) A^{2}} A f(X(s))\right\| \mathrm{d} s \\
& \leq\left|X_{0}\right|_{3}+C \int_{0}^{t}(t-s)^{-\frac{3}{4}}\|A f(X(s))\| \mathrm{d} s .
\end{aligned}
$$

We apply 2.17) to $\|A f(X(s))\|=\|\Delta f(X(s))\|$ to get

$$
\begin{aligned}
|Y(t)|_{3} & \leq\left|X_{0}\right|_{3}+C \int_{0}^{t}(t-s)^{-\frac{3}{4}}\left(1+\|X(s)\|_{1}^{2}\right)\|X(s)\|_{3} \mathrm{~d} s \\
& \leq\left|X_{0}\right|_{3}+C \int_{0}^{t}(t-s)^{-\frac{3}{4}}\left(1+\|X(s)\|_{1}^{2}\right)\left(\|Y(s)\|_{3}+\left\|W_{A}(s)\right\|_{3}\right) \mathrm{d} s .
\end{aligned}
$$

Since $(I-P) Y(t)=(I-P) X_{0}$ is constant, we get the same bound for the norm $\|Y(t)\|_{3}$. Using also (4.4) gives

$$
\begin{aligned}
\|Y(t)\|_{3} \leq & \left\|X_{0}\right\|_{3}+C \int_{0}^{t}(t-s)^{-\frac{3}{4}}\left(1+\epsilon^{-1} K_{T}\right)\left(\|Y(s)\|_{3}+\epsilon^{-1} K_{T}\right) \mathrm{d} s \\
\leq & \left\|X_{0}\right\|_{3}+C \epsilon^{-1} K_{T}\left(1+\epsilon^{-1} K_{T}\right) T^{\frac{1}{4}} \\
& +C\left(1+\epsilon^{-1} K_{T}\right) \int_{0}^{t}(t-s)^{-\frac{3}{4}}\|Y(s)\|_{3} \mathrm{~d} s .
\end{aligned}
$$


Applying Gronwall's Lemma 2.3 with $\alpha=1, \beta=\frac{1}{4}$ and

$$
A=\left\|X_{0}\right\|_{3}+C \epsilon^{-1} K_{T}\left(1+\epsilon^{-1} K_{T}\right), B=C\left(1+\epsilon^{-1} K_{T}\right),
$$

gives

$$
\|Y(t)\|_{3} \leq A C(B, T)=C\left(\left\|X_{0}\right\|_{3}, \epsilon^{-1} K_{T}, T\right), \quad t \in[0, T] .
$$

The bound for $\|X(t)\|_{3}$ then follows in view of (4.4).

The constant $C\left(\left\|X_{0}\right\|_{3}, \epsilon^{-1} K_{T}, T\right)$ grows rapidly with $\epsilon^{-1} K_{T}$ and $T$. Hence, it is important that $K_{T}$ grows only quadratically with $T$. Also note that the proof of Theorem 4.3 shows that under the assumptions of the theorem, in fact, $f(X(t)) \in$ $D(A)$ almost surely and $\|A f(X(t))\|<\infty$ almost surely for $t \in[0, T]$. Therefore, $X$ satisfies a more strict (in comparison to (3.5) ) mild form of (3.3):

$$
X(t)=\mathrm{e}^{-t A^{2}} X_{0}-\int_{0}^{t} \mathrm{e}^{-(t-s) A^{2}} A f(X(s)) \mathrm{d} s+\int_{0}^{t} \mathrm{e}^{-(t-s) A^{2}} \mathrm{~d} W(s) .
$$

\section{Error estimates.}

5.1. The linear deterministic Cahn-Hilliard equation. Consider the linear Cahn-Hilliard equation

$$
\dot{u}+A v=0, v-A u-f=0, t>0 ; u(0)=u_{0},
$$

where $f$ is a function of $x, t$, and the corresponding finite element problem

$$
\dot{u}_{h}+A_{h} v_{h}=0, v_{h}-A_{h} u_{h}-P_{h} f=0, t>0 ; u_{h}(0)=P_{h} u_{0} .
$$

We have the following error estimate. We will later use this for fixed $\omega \in \Omega_{\epsilon}$ with $f$ replaced by $f(X)$ and $u$ by the solution $Y$ of (4.1). The error estimate differs from the corresponding error estimates in [7, 12] in that it contains no time derivative. This is important since $Y$ has limited temporal regularity.

THEOREM 5.1. Assume that $u, v$ and $u_{h}, v_{h}$ are weak solutions of (5.1) and (5.2), respectively. Then, for $t \geq 0$ and $h \in\left(0, \frac{1}{2}\right]$, we have

$$
\left\|u_{h}(t)-u(t)\right\| \leq C h^{2}\left(|\log (h)| \max _{0 \leq s \leq t}|u(s)|_{2}+\left(\int_{0}^{t}|v(s)|_{2}^{2} \mathrm{~d} s\right)^{\frac{1}{2}}\right) .
$$

Proof. The weak forms of (5.1) and $(5.2)$ are

$$
\begin{array}{ll}
\left\langle\dot{u}, \varphi_{1}\right\rangle+\left\langle\nabla v, \nabla \varphi_{1}\right\rangle=0 & \forall \varphi_{1} \in H^{1} \\
\left\langle v, \varphi_{2}\right\rangle-\left\langle\nabla u, \nabla \varphi_{2}\right\rangle-\left\langle f, \varphi_{2}\right\rangle=0 & \forall \varphi_{2} \in H^{1}, \\
u(0)=u_{0}, &
\end{array}
$$

and

$$
\begin{array}{ll}
\left\langle\dot{u}_{h}, \varphi_{h, 1}\right\rangle+\left\langle\nabla v_{h}, \nabla \varphi_{h, 1}\right\rangle=0 & \forall \varphi_{h, 1} \in S_{h}, \\
\left\langle v_{h}, \varphi_{h, 2}\right\rangle-\left\langle\nabla u_{h}, \nabla \varphi_{h, 2}\right\rangle-\left\langle f, \varphi_{h, 2}\right\rangle=0 & \forall \varphi_{h, 2} \in S_{h}, \\
u_{h}(0)=P_{h} u_{0} . &
\end{array}
$$


Let $P_{h}$ and $R_{h}$ be as in 2.7$)$ and 2.10 and set

$$
\begin{aligned}
& e_{u}=u_{h}-u=\left(u_{h}-P_{h} u\right)+\left(P_{h} u-u\right)=\theta_{u}+\rho_{u}, \\
& e_{v}=v_{h}-v=\left(v_{h}-R_{h} v\right)+\left(R_{h} v-v\right)=\theta_{v}+\rho_{v} .
\end{aligned}
$$

We want to compute

$$
\left\|e_{u}\right\| \leq\left\|\theta_{u}\right\|+\left\|\rho_{u}\right\| .
$$

In (5.4 choose $\varphi_{1}=\varphi_{h, 1}$ and $\varphi_{2}=\varphi_{h, 2}$ and subtract the first two equations of (5.4) from the corresponding equations in 5.5 to get

$$
\begin{array}{ll}
\left\langle\dot{e}_{u}, \varphi_{h, 1}\right\rangle+\left\langle\nabla e_{v}, \nabla \varphi_{h, 1}\right\rangle=0 & \forall \varphi_{h, 1} \in S_{h}, \\
\left\langle e_{v}, \varphi_{h, 2}\right\rangle-\left\langle\nabla e_{u}, \nabla \varphi_{h, 2}\right\rangle=0 & \forall \varphi_{h, 2} \in S_{h} .
\end{array}
$$

Hence, by (5.6) and (5.7),

$$
\begin{aligned}
& \left\langle\dot{\theta}_{u}, \varphi_{h, 1}\right\rangle+\left\langle\nabla \theta_{v}, \nabla \varphi_{h, 1}\right\rangle=-\left\langle\dot{\rho}_{u}, \varphi_{h, 1}\right\rangle-\left\langle\nabla \rho_{v}, \nabla \varphi_{h, 1}\right\rangle \quad \forall \varphi_{h, 1} \in S_{h}, \\
& \left\langle\theta_{v}, \varphi_{h, 2}\right\rangle-\left\langle\nabla \theta_{u}, \nabla \varphi_{h, 2}\right\rangle=-\left\langle\rho_{v}, \varphi_{h, 2}\right\rangle+\left\langle\nabla \rho_{u}, \nabla \varphi_{h, 2}\right\rangle \quad \forall \varphi_{h, 2} \in S_{h} .
\end{aligned}
$$

By the definitions of $P_{h}$ and $R_{h}$ we have

$$
\begin{aligned}
\left\langle\dot{\rho}_{u}, \varphi_{h, 1}\right\rangle & =\left\langle P_{h} \dot{u}-\dot{u}, \varphi_{h, 1}\right\rangle=0 & & \forall \varphi_{h, 1} \in S_{h}, \\
\left\langle\nabla \rho_{v}, \nabla \varphi_{h, 1}\right\rangle & =\left\langle\nabla R_{h} v-v, \nabla \varphi_{h, 1}\right\rangle=0 & & \forall \varphi_{h, 2} \in S_{h},
\end{aligned}
$$

so that

$$
\begin{aligned}
& \left\langle\dot{\theta}_{u}, \varphi_{h, 1}\right\rangle+\left\langle\nabla \theta_{v}, \nabla \varphi_{h, 1}\right\rangle=0 \quad \forall \varphi_{h, 1} \in S_{h}, \\
& \left\langle\theta_{v}, \varphi_{h, 2}\right\rangle-\left\langle\nabla \theta_{u}, \nabla \varphi_{h, 2}\right\rangle=-\left\langle P_{h} \rho_{v}, \varphi_{h, 2}\right\rangle+\left\langle\nabla R_{h} \rho_{u}, \nabla \varphi_{h, 2}\right\rangle \quad \forall \varphi_{h, 2} \in S_{h} .
\end{aligned}
$$

In the second equation we set $\varphi_{h, 2}=A_{h} \varphi_{h, 1}$ to get

$$
\left\langle\nabla \theta_{v}, \nabla \varphi_{h, 1}\right\rangle=\left\langle A_{h}^{2} \theta_{u}, \varphi_{h, 1}\right\rangle-\left\langle A_{h} P_{h} \rho_{v}, \varphi_{h, 1}\right\rangle+\left\langle A_{h}^{2} R_{h} \rho_{u}, \varphi_{h, 1}\right\rangle .
$$

Inserting this into the first equation gives

$$
\left\langle\dot{\theta}_{u}, \varphi_{h, 1}\right\rangle+\left\langle A_{h}^{2} \theta_{u}, \varphi_{h, 1}\right\rangle=\left\langle A_{h} P_{h} \rho_{v}, \varphi_{h, 1}\right\rangle-\left\langle A_{h}^{2} R_{h} \rho_{u}, \varphi_{h, 1}\right\rangle,
$$

so the strong form is

$$
\dot{\theta}_{u}+A_{h}^{2} \theta_{u}=A_{h} P_{h} \rho_{v}-A_{h}^{2} R_{h} \rho_{u}, \quad t>0 ; \quad \theta_{u}(0)=0,
$$

with solution

$$
\theta_{u}(t)=\int_{0}^{t} \mathrm{e}^{-(t-s) A_{h}^{2}} A_{h} P_{h} \rho_{v}(s) \mathrm{d} s-\int_{0}^{t} \mathrm{e}^{-(t-s) A_{h}^{2}} A_{h}^{2} R_{h} \rho_{u}(s) \mathrm{d} s .
$$

Taking norms here gives

$$
\begin{aligned}
\left\|\theta_{u}(t)\right\| \leq & \left\|\int_{0}^{t} \mathrm{e}^{-(t-s) A_{h}^{2}} A_{h} P_{h} \rho_{v}(s) \mathrm{d} s\right\| \\
& +\left\|\int_{0}^{t} \mathrm{e}^{-(t-s) A_{h}^{2}} A_{h}^{2} R_{h} \rho_{u}(s) \mathrm{d} s\right\|=I+I I .
\end{aligned}
$$


For $I$ we define

$$
w_{h}(t)=\int_{0}^{t} \mathrm{e}^{-(t-s) A_{h}^{2}} P_{h} \rho_{v}(s) \mathrm{d} s
$$

which satisfies the equation

$$
\dot{w}_{h}+A_{h}^{2} w_{h}=P_{h} \rho_{v}, \quad t>0 ; \quad w_{h}(0)=0 .
$$

We multiply by $\dot{w}_{h}$ to get

$$
\left\|\dot{w}_{h}\right\|^{2}+\frac{1}{2} \frac{\mathrm{d}}{\mathrm{d} t}\left\|A_{h} w_{h}\right\|^{2}=\left\langle P_{h} \rho_{v}, \dot{w}_{h}\right\rangle \leq\left\|\rho_{v}\right\|\left\|\dot{w}_{h}\right\| \leq \frac{1}{2}\left\|\rho_{v}\right\|^{2}+\frac{1}{2}\left\|\dot{w}_{h}\right\|^{2},
$$

so that

$$
\left\|\dot{w}_{h}\right\|^{2}+\frac{\mathrm{d}}{\mathrm{d} t}\left\|A_{h} w_{h}\right\|^{2} \leq\left\|\rho_{v}\right\|^{2}
$$

Integration and ignoring $\int_{0}^{t}\left\|\dot{w}_{h}(s)\right\|^{2} \mathrm{~d} s$ leads to

$$
\left\|A_{h} \int_{0}^{t} \mathrm{e}^{-(t-s) A_{h}^{2}} P_{h} \rho_{v}(s) \mathrm{d} s\right\|=\left\|A_{h} w_{h}(t)\right\| \leq\left(\int_{0}^{t}\left\|\rho_{v}(s)\right\|^{2} \mathrm{~d} s\right)^{\frac{1}{2}},
$$

where, from 2.11,

$$
\left\|\rho_{v}\right\|=\left\|\left(R_{h}-I\right) v\right\| \leq C h^{2}|v|_{2} .
$$

Hence,

$$
\left\|A_{h} \int_{0}^{t} \mathrm{e}^{-(t-s) A_{h}^{2}} P_{h} \rho_{v}(s) \mathrm{d} s\right\| \leq C h^{2}\left(\int_{0}^{t}|v(s)|_{2}^{2} \mathrm{~d} s\right)^{\frac{1}{2}} .
$$

For the term $I I$ we use

$$
R_{h} \rho_{u}=R_{h}\left(P_{h} u-u\right)=P_{h} u-R_{h} u=P_{h}\left(u-R_{h} u\right) .
$$

Then

$$
\begin{gathered}
\left\|\int_{0}^{t} A_{h}^{2} \mathrm{e}^{-(t-s) A_{h}^{2}} R_{h} \rho_{u}(s) \mathrm{d} s\right\| \leq \int_{0}^{t}\left\|A_{h}^{2} \mathrm{e}^{-(t-s) A_{h}^{2}} P_{h}\left(u(s)-R_{h} u(s)\right)\right\| \mathrm{d} s \\
\quad \leq \int_{0}^{t}\left\|A_{h}^{2} \mathrm{e}^{-(t-s) A_{h}^{2}} P_{h}\right\| \mathrm{d} s \max _{0 \leq s \leq t}\left\|u(s)-R_{h} u(s)\right\| .
\end{gathered}
$$

Here we use $\left\|A_{h}\right\| \leq C h^{-2}$ from 2.12 and 2.9 to get

$$
\begin{gathered}
\int_{0}^{t}\left\|A_{h}^{2} \mathrm{e}^{-(t-s) A_{h}^{2}} P_{h} P\right\| \mathrm{d} s=\int_{0}^{h^{4}}\left\|A_{h}\right\|^{2}\left\|\mathrm{e}^{-s A_{h}^{2}}\right\| \mathrm{d} s+\int_{h^{4}}^{t}\left\|A_{h}^{2} \mathrm{e}^{-s A_{h}^{2}}\right\| \mathrm{d} s \\
\leq C h^{-4} h^{4}+C \int_{h^{4}}^{t} s^{-1} \mathrm{e}^{-c s} \mathrm{~d} s \leq C(1+\log (1 / h)) \leq C|\log (h)|
\end{gathered}
$$

for $h \in\left(0, \frac{1}{2}\right]$. Hence, by 2.11, we have

$$
\left\|\int_{0}^{t} A_{h}^{2} \mathrm{e}^{-(t-s) A_{h}^{2}} R_{h} \rho_{u}(s) \mathrm{d} s\right\| \leq C h^{2}|\log (h)| \max _{0 \leq s \leq t}|u(s)|_{2} .
$$


Inserting (5.10) and 5.11) into 5.9 gives

$$
\left\|\theta_{u}(t)\right\| \leq C h^{2}\left\{\left(\int_{0}^{t}|v(s)|_{2}^{2} \mathrm{~d} s\right)^{\frac{1}{2}}+|\log (h)| \max _{0 \leq s \leq t}|u(s)|_{2}\right\} .
$$

Finally, by the best approximation property of $P_{h}$,

$$
\left\|\rho_{u}(t)\right\|=\left\|P_{h} u-u\right\| \leq\left\|R_{h} u-u\right\| \leq C h^{2}|u(t)|_{2} .
$$

Inserting (5.12) and (5.13) into (5.8) gives the desired result (5.3).

The following regularity estimate for the linear Cahn-Hilliard equation (5.1) is proved by an elementary energy argument.

Lemma 5.2. Assume that $u, v$ are weak solutions of (5.1). Then

$$
|u(t)|_{2}^{2}+\int_{0}^{t}|v(s)|_{2}^{2} \mathrm{~d} s \leq\left|u_{0}\right|_{2}^{2}+\int_{0}^{t}|f(s)|_{2}^{2} \mathrm{~d} s
$$

5.2. Error estimate for the stochastic Cahn-Hilliard equation. In the next theorem we prove an error estimate for the nonlinear Cahn-Hilliard-Cook equation.

TheOREM 5.3. Assume that $\left\|A^{\frac{\gamma}{2}} Q^{\frac{1}{2}}\right\|_{\mathrm{HS}}<\infty$ for some $\gamma>1$ and that $X_{0}$ is $\mathcal{F}_{0}$-measurable with values in $H^{3}$ satisfying $\left\|X_{0}\right\|_{L_{2}\left(\Omega, H^{1}\right)}^{2}+\left\|X_{0}\right\|_{L_{4}\left(\Omega, L_{4}\right)}^{4} \leq \rho$ for some $\rho \geq 0$. Let $T>0, \epsilon \in(0,1)$, and let $\Omega_{\epsilon} \subset \Omega$ and $K_{T}$ be as in Corollary 3.2 such that also $\left\|X_{0}\right\|_{3} \leq C$ on $\Omega_{\epsilon}$. If $X$ is the weak solution of (3.3) and $X_{h}$ is the solution of (3.6), then, for $h \in\left(0, \frac{1}{2}\right]$,

$$
\left\|X_{h}(t)-X(t)\right\| \leq C\left(\left\|X_{0}\right\|_{3}, \epsilon^{-1} K_{T}, T\right) h^{2}|\log (h)|, \quad \text { on } \Omega_{\epsilon}, t \in[0, T] .
$$

The constant $C\left(\left\|X_{0}\right\|_{3}, \epsilon^{-1} K_{T}, T\right)$ grows rapidly with $\epsilon^{-1} K_{T}$ and $T$ due to the use of Gronwall's lemma in the proof. As noted before in the proof of Theorem 4.3 . it is always possible to choose $\Omega_{\epsilon}$ in Corollary 3.2 such that also $\left\|X_{0}\right\|_{3} \leq C$ on $\Omega_{\epsilon}$.

Proof. Let $\omega \in \Omega_{\epsilon}$ be fixed. Set

$$
X(t)=Y(t)+W_{A}(t),
$$

where $W_{A}(t)$ is the stochastic convolution 2.14 and $Y(t)$ is the weak solution 4.5 of 4.1. Also set

$$
X_{h}(t)=Z_{h}(t)+W_{A_{h}}(t),
$$

where $W_{A_{h}}(t)$ is the stochastic convolution 2.15 and

$$
Z_{h}(t)=\mathrm{e}^{-t A_{h}^{2}} P_{h} X_{0}-\int_{0}^{t} \mathrm{e}^{-(t-s) A_{h}^{2}} A_{h} P_{h} f\left(X_{h}(s)\right) \mathrm{d} s .
$$

Finally, let

$$
Y_{h}(t)=\mathrm{e}^{-t A_{h}^{2}} P_{h} X_{0}-\int_{0}^{t} \mathrm{e}^{-(t-s) A_{h}^{2}} A_{h} P_{h} f(X(s)) \mathrm{d} s .
$$

We subtract 5.14 from 5.15 and take norms,

$$
\left\|X_{h}-X\right\| \leq\left\|W_{A_{h}}-W_{A}\right\|+\left\|Y_{h}-Y\right\|+\left\|Z_{h}-Y_{h}\right\| .
$$


We must compute the three norms on the right-hand side.

First we compute $\left\|W_{A_{h}}(t)-W_{A}(t)\right\|$. Since $\left\|A^{\frac{1}{2}} Q^{\frac{1}{2}}\right\|_{\text {HS }}<\infty$, we have that $\left\|Q^{\frac{1}{2}}\right\|_{\mathrm{HS}}<\infty$, see 3.19 , and hence, by Theorem 2.2 and Chebyshev's inequality, we get

$$
\begin{aligned}
\left\|W_{A_{h}}(t)-W_{A}(t)\right\| & \leq \epsilon^{-\frac{1}{2}}\left(\mathbf{E}\left[\left\|W_{A_{h}}(t)-W_{A}(t)\right\|^{2}\right]\right)^{\frac{1}{2}} \\
& \leq \epsilon^{-\frac{1}{2}} C h^{2}|\log (h)|\left\|Q^{\frac{1}{2}}\right\|_{\mathrm{HS}} \leq C\left(\epsilon^{-1} K_{Q}\right)^{\frac{1}{2}} h^{2}|\log (h)|,
\end{aligned}
$$

where $K_{Q}$ is as in Theorem 3.1 Since $K_{Q} \leq K_{T}$, we conclude

$$
\left\|W_{A_{h}}(t)-W_{A}(t)\right\| \leq C\left(\epsilon^{-1} K_{T}\right)^{\frac{1}{2}} h^{2}|\log (h)| .
$$

Now we consider $\left\|Y_{h}(t)-Y(t)\right\|$ and use Theorem 5.1 to get

$$
\left\|Y_{h}(t)-Y(t)\right\| \leq C h^{2}\left\{|\log (h)| \max _{0 \leq s \leq t}|Y(s)|_{2}+\left(\int_{0}^{t}|V(s)|_{2}^{2} \mathrm{~d} s\right)^{\frac{1}{2}}\right\}
$$

where $Y(t)$ and $V(t)$ are the solutions of

$$
\dot{Y}+A V=0, V=A Y+f(X), t \in(0, T] ; Y(0)=X_{0} .
$$

By using Lemma 5.2 (2.17), 3.25), and Theorem 4.3 , we get

$$
\begin{aligned}
\int_{0}^{t}|V(s)|_{2}^{2} \mathrm{~d} s & \leq\left|X_{0}\right|_{2}^{2}+\int_{0}^{t}|f(X(s))|_{2}^{2} \mathrm{~d} s \\
& \leq\left\|X_{0}\right\|_{2}^{2}+C \int_{0}^{t}\left(1+\|X(s)\|_{1}^{2}\right)^{2}\|X(s)\|_{3}^{2} \mathrm{~d} s \\
& \leq C\left(\left\|X_{0}\right\|_{3}, \epsilon^{-1} K_{T}, T\right) .
\end{aligned}
$$

Now we bound $|Y(t)|_{2}$. By Theorem 4.3 we have

$$
|Y(t)|_{2} \leq\|Y(t)\|_{3} \leq C\left(\left\|X_{0}\right\|_{3}, \epsilon^{-1} K_{T}, T\right) .
$$

Using 5.21 and 5.22 in 5.20 gives

$$
\left\|Y_{h}(t)-Y(t)\right\| \leq C\left(\left\|X_{0}\right\|_{3}, \epsilon^{-1} K_{T}, T\right) h^{2}|\log (h)| .
$$

Finally we compute $\left\|e_{h}(t)\right\|=\left\|Z_{h}(t)-Y_{h}(t)\right\|$. By subtraction of (5.16) and (5.17), we obtain

$$
\begin{aligned}
\left\|e_{h}(t)\right\| & \leq \int_{0}^{t}\left\|\mathrm{e}^{-(t-s) A_{h}^{2}} A_{h} P_{h} P\left(f\left(X_{h}(s)\right)-f(X(s))\right)\right\| \mathrm{d} s \\
& \leq \int_{0}^{t}\left\|A_{h}^{\frac{3}{2}} \mathrm{e}^{-(t-s) A_{h}^{2}}\right\|\left\|A_{h}^{-\frac{1}{2}} P\left(f\left(X_{h}(s)\right)-f(X(s))\right)\right\| \mathrm{d} s,
\end{aligned}
$$

since the constant eigenmodes cancel (cf. (2.16)). Using $(2.18)$ and 2.9 gives

$$
\left\|e_{h}(t)\right\| \leq C \int_{0}^{t}(t-s)^{-\frac{3}{4}}\left(1+\left\|X_{h}(s)\right\|_{1}^{2}+\|X(s)\|_{1}^{2}\right)\left\|X_{h}(s)-X(s)\right\| \mathrm{d} s .
$$


By Corollary 3.2 we have

$$
\begin{aligned}
\left\|e_{h}(t)\right\| \leq & C \int_{0}^{t}(t-s)^{-\frac{3}{4}}\left(1+\epsilon^{-1} K_{T}\right)\left(\left\|W_{A_{h}}(s)-W_{A}(s)\right\|\right. \\
& \left.+\left\|Y_{h}(s)-Y(s)\right\|+\left\|e_{h}(s)\right\|\right) \mathrm{d} s \\
\leq & C\left(1+\epsilon^{-1} K_{T}\right) T^{\frac{1}{4}} \max _{0 \leq s \leq T}\left(\left\|W_{A_{h}}(s)-W_{A}(s)\right\|+\left\|Y_{h}(s)-Y(s)\right\|\right) \\
& +C\left(1+\epsilon^{-1} K_{T}\right) \int_{0}^{t}(t-s)^{-\frac{3}{4}}\left\|e_{h}(s)\right\| \mathrm{d} s .
\end{aligned}
$$

We apply Gronwall's Lemma 2.3 with $\alpha=1, \beta=\frac{1}{4}$ and

$$
\begin{aligned}
& A=C\left(1+\epsilon^{-1} K_{T}\right) T^{\frac{1}{4}} \max _{0 \leq s \leq T}\left(\left\|W_{A_{h}}(s)-W_{A}(s)\right\|+\left\|Y_{h}(s)-Y(s)\right\|\right), \\
& B=C\left(1+\epsilon^{-1} K_{T}\right),
\end{aligned}
$$

to get

$$
\left\|Z_{h}(t)-Y_{h}(t)\right\|=\left\|e_{h}(t)\right\| \leq A C(B, T), \quad t \in[0, T] .
$$

But we already obtained bounds for $\left\|W_{A_{h}}(t)-W_{A}(t)\right\|$ and $\left\|Y_{h}(t)-Y(t)\right\|$ in (5.19) and (5.23). By inserting these and (5.24) into (5.18) we get the desired result.

Since we have regularity of order 3 on $\Omega_{\epsilon}$, it would be possible to prove convergence of order 3 for piecewise quadratic finite elements. We do not find this worth the extra effort since our main result does not show a rate of convergence anyway.

We finally show that $X_{h}$ converges strongly to $X$.

THEOREM 5.4. Assume that $\left\|A^{\frac{\gamma}{2}} Q^{\frac{1}{2}}\right\|_{\mathrm{HS}}<\infty$ for some $\gamma>1$ and that $X_{0}$ is $\mathcal{F}_{0}$-measurable with values in $H^{3}$ satisfying $\left\|X_{0}\right\|_{L_{2}\left(\Omega, H^{1}\right)}^{2}+\left\|X_{0}\right\|_{L_{4}\left(\Omega, L_{4}\right)}^{4} \leq \rho$ for some $\rho \geq 0$. If $X$ is the weak solution of (3.3) and $X_{h}$ is the solution of 3.6 , then

$$
\left(\mathbf{E}\left[\sup _{t \in[0, T]}\left\|X_{h}(t)-X(t)\right\|^{2}\right]\right)^{\frac{1}{2}} \rightarrow 0 \quad \text { as } h \rightarrow 0 .
$$

Proof. From Corollary 3.2 it follows that

$$
\mathbf{E}\left[\sup _{t \in[0, T]}\|X(t)\|_{L_{4}}^{4}\right] \leq K_{T}, \quad \mathbf{E}\left[\sup _{t \in[0, T]}\left\|X_{h}(t)\right\|_{L_{4}}^{4}\right] \leq K_{T}, \quad t \in[0, T],
$$

with $K_{T}$ as in Corollary 3.2 . Let $\epsilon \in(0,1)$ and let $\Omega_{\epsilon}$ be as in Corollary 3.2 such that also $\left\|X_{0}\right\|_{3} \leq C$ on $\Omega_{\epsilon}$. Then

$$
\begin{aligned}
\mathbf{E}\left[\sup _{t \in[0, T]}\left\|X_{h}(t)-X(t)\right\|^{2}\right] \leq & \int_{\Omega_{\epsilon}} \sup _{t \in[0, T]}\left\|X_{h}(t)-X(t)\right\|^{2} \mathrm{~d} \mathbf{P} \\
& +2 \int_{\Omega_{\epsilon}^{c}}\left(\sup _{t \in[0, T]}\left\|X_{h}(t)\right\|^{2}+\sup _{t \in[0, T]}\|X(t)\|^{2}\right) \mathrm{d} \mathbf{P} .
\end{aligned}
$$

Here, by Hölder's inequality and (5.25), we have

$$
\begin{aligned}
\int_{\Omega_{\epsilon}^{c}} \sup _{t \in[0, T]}\|X(t)\|^{2} \mathrm{~d} \mathbf{P} & \leq\left(\int_{\Omega_{\epsilon}^{c}} 1^{2} \mathrm{~d} \mathbf{P}\right)^{\frac{1}{2}}\left(\int_{\Omega_{\epsilon}^{c}} \sup _{t \in[0, T]}\|X(t)\|_{L_{4}}^{4} \mathrm{~d} \mathbf{P}\right)^{\frac{1}{2}} \\
& \leq \epsilon^{\frac{1}{2}}\left(\mathbf{E}\left[\sup _{t \in[0, T]}\|X(t)\|_{L_{4}}^{4}\right]\right)^{\frac{1}{2}} \leq \epsilon^{\frac{1}{2}} K_{T}^{\frac{1}{2}}
\end{aligned}
$$


and similarly for $X_{h}$. Therefore, by Theorem 5.3 .

$$
\left(\mathbf{E}\left[\sup _{t \in[0, T]}\left\|X_{h}(t)-X(t)\right\|^{2}\right]\right)^{\frac{1}{2}} \leq C\left(\epsilon^{-1} K_{T}, T\right) h^{2}|\log (h)|+C K_{T}^{\frac{1}{4}} \epsilon^{\frac{1}{4}} .
$$

Since $\frac{\epsilon^{\frac{1}{4}}}{C\left(\epsilon^{-1} K_{T}, T\right)} \rightarrow 0$ monotonically as $\epsilon \rightarrow 0$, we may choose $\epsilon$ depending on $h$, such that the two terms are equal.

Since $C\left(\epsilon^{-1} K_{T}, T\right)$ grows rapidly with $\epsilon^{-1}$, is not possible to obtain a rate of convergence from this proof.

Acknowledgments. The authors thank the anonymous referees for their valuable suggestions which helped to improve the quality of the paper.

\section{REFERENCES}

[1] J. M. BALL, Strongly continuous semigroups, weak solutions, and the variation of constants formula, Proc. Amer. Math. Soc., 63 (1977), pp. 370-373.

[2] D. Blömker, S. Maier-PaApe, And T. Wanner, Second phase spinodal decomposition for the Cahn-Hilliard-Cook equation, Trans. Amer. Math. Soc., 360 (2008), pp. 449-489.

[3] C. Cardon-Weber, Implicit approximation scheme for the Cahn-Hilliard stochastic equation, Preprint, Laboratoire des Probabilités et Modelèles Aléatoires, Université Paris VI, 2000, http://citeseer.ist.psu.edu/633895.html.

[4] H. E. Cook, Brownian motion in spinodal decomposition, Acta Metallurgica, 18 (1970), pp. 297-306.

[5] G. Da Prato and A. Debussche, Stochastic Cahn-Hilliard equation, Nonlinear Anal., 26 (1996), pp. 241-263.

[6] G. Da Prato And J. Zabczyk, Stochastic Equations in Infinite Dimensions, Cambridge University Press, Cambridge, 1992.

[7] C. M. Elliott and S. Larsson, Error estimates with smooth and nonsmooth data for a finite element method for the Cahn-Hilliard equation, Math. Comp., 58 (1992), pp. 603-630, S33-S36.

[8] D. Henry, Geometric Theory of Semilinear Parabolic Equations, Lecture Notes in Mathematics, vol. 840, Springer-Verlag, Berlin-New York, 1981.

[9] D. J. Higham, X. MaO, and A. M. Stuart, Strong convergence of Euler-type methods for nonlinear stochastic differential equations, SIAM J. Numer. Anal., 40 (2002), pp. 10411063.

[10] G. T. Kossioris And G. E. Zouraris, Fully-discrete finite element approximations for a fourthorder linear stochastic parabolic equation with additive space-time white noise, M2AN Math. Model. Numer. Anal., 44 (2010), pp. 289-322.

[11] M. Kovács, S. Larsson, ANd F. Lindgren, Weak convergence of finite element approximations of linear stochastic evolution equations with additive noise, BIT Numer. Math. (2011), to appear. Doi: 10.1007/s10543-011-0344-2.

[12] S. LARsson And A. Mesforush, Finite element approximation of the linearized Cahn-HilliardCook equation, IMA J. Numer. Anal. (2011), to appear. Doi: 10.1093/imanum/drq042.

[13] V. Thomée, Galerkin Finite Element Methods for Parabolic Problems, Second ed., Springer Series in Computational Mathematics, vol. 25, Springer-Verlag, Berlin, 2006. 